\title{
Identification of Vibration Modes of Quartz Crystal Plates with Proportion of Strain and Kinetic Energies
}

\author{
Qi Huang \\ Piezoelectric Device Laboratory, School of Mechanical Engineering and Mechanics, Ningbo University,818 \\ Fenghua Road, Ningbo, Zhejiang 315211, China. \\ Department of Architectural Engineering, Huzhou Vocational \& Technical College, 299 Xuefu Road, Wuxing Dis- \\ trict, Huzhou, Zhejiang 313000, China.
}

\begin{abstract}
Rongxing Wu
Department of Architectural Engineering, Ningbo Polytechnic, 1069 Xinda Road, Beilun District, Ningbo, Zhejiang 315800, China.
\end{abstract}

\author{
Lihong Wang, Longtao Xie, Jianke Du, Tingfeng Ma and Ji Wang ${ }^{\dagger}$ \\ Piezoelectric Device Laboratory, School of Mechanical Engineering and Mechanics, Ningbo University,818 \\ Fenghua Road, Ningbo, Zhejiang 315211, China. \\ $\dagger$ Corresponding author
}

(Received 5 November 2019; accepted 18 May 2020)

For the design of quartz crystal resonators, finding and determining the vibration modes have always been very important and cumbersome. Vibration modes are usually identified through plotting displacement patterns of each coupled modes and making comparisons. Over the years, there is not much improvement in the identification procedure while tremendous efforts have been made in refining the equations of the Mindlin plate theory to obtain more accurate results, such as the adoption of the Finite Element Method (FEM) by implementing the high-order Mindlin plate equations for efficient analysis. However, due to the old fashioned mode identification method, the FEM application is still inadequate and cannot be fully automated for this purpose. To have this situation improved, a method using the proportions of strain and kinetic energies to characterize the energy level of each vibration mode is proposed. With solutions of displacements, the energy distribution of each vibration mode is calculated and the mode with the highest energy concentration at a specific frequency is designated as the dominant mode. The results have been validated with the traditional approach by plotting mode shapes at each frequency. Clearly, this energy approach will be advantageous with the FEM analysis for vibration mode identification automatically.

\section{INTRODUCTION}

In the design of a quartz crystal resonator, it is important to make full and accurate analysis of its vibrations and to identify the functioning mode through the detailed results like frequency and mode shapes. ${ }^{1-4}$ The precise identification of vibration modes is essential because the optimal functioning mode of the resonator in the designated vibration frequency should be dominant and needs to be well isolated from so-called spurious modes of negative effects. Consequently, the functioning mode is extremely important to be identified, which is the thickness-shear mode usually, to immunize from couplings to other modes, or the notorious spurious modes. As there is no suitable way to eliminate the infinite couplings of vibration modes in a finite quartz crystal plate, it is practical to find the least coupled thickness-shear vibrations to enhance the performance characteristics of a quartz crystal resonator. In other words, the challenge is to determine the optimal parameters of the resonator structure, avoid the strong coupling of modes, and let the primary working mode be enhanced. ${ }^{3}$ In a typical resonator, the primary working mode mainly refers to the thickness-shear mode and the strongly coupled one is the flexural mode. ${ }^{2}$ For the accurate analysis of vibrations within a quartz crystal plate in the vicinity of the thickness-shear mode, the most effective method we have chosen is based on the Mindlin plate theory which can easily include more vibration modes.

The strong couplings happen just between the neighboring vibration modes, so only a few strongly coupled vibration modes are needed for the analysis to obtain the accurate results such as frequency and displacements which can be used for mode identification. Usually the analysis is done with the straight-crested waves for applications, and currently the Mindlin plate equations have been implemented in the finite element analysis for more precise considerations of structural complications. The identification of vibration modes, as from the beginning, is done with the plotting of mode shapes which show the dominance of specific mode at each frequency by picking out the largest displacement. ${ }^{4,5}$ 
Initially, the analysis of plate vibrations was presented as a three-dimensional problem ${ }^{6}$ which inevitably demanded complicated calculations in solving the differential equations of motion. Fortunately, with the Mindlin plate theory, we can analyze the vibrations from a two-dimensional perspective and this greatly simplifies the solution procedure. Accordingly, accurate solutions from the Mindlin plate equations with analytical and numerical procedures have made considerable progress recently corresponding with methods to enhance the mode identification, and in conjunction with the finite element method, it is highly regarded as a major improvement. ${ }^{7}$ As a result, the mode shapes can be plotted and identified with the displacements.

In the early beginning, the Mindlin plate theory was presented with only equations of zeroth- and first-order mechanical displacements, ${ }^{8}$ then Mindlin and Tiersten ${ }^{9}$ expanded the two-dimensional equations of the plate under forced vibrations to higher-order equations, and introduced the electric field considerations. Later Mindlin and Spencer ${ }^{2}$ presented the normalized wavenumber and dispersion relations of the infinite plate. Furthermore, equations of AT-cut quartz crystal rectangular plates in free vibrations were derived and the frequency spectra were calculated. ${ }^{2}$ The normalized dispersion relations and frequency spectra, as effective representations of the vibration analysis results, were widely used for the optimal selection of plate parameters. Of course, other researchers also proposed similar or novel two-dimensional theory in resonator vibration analysis, like Lee, et al. ${ }^{10}$ and Sekimoto, et al. ${ }^{11}$

In recent years, applications of Mindlin plate theory have been further improved. Based on the high-order Mindlin plate theory, Yong and Zhang ${ }^{12}$ and Wang, et al. ${ }^{13}$ developed the finite element method in analyzing high-frequency vibrations of quartz crystal resonators. Then Yong, et al. ${ }^{14}$ studied vibration modes with the first- and third-order plate equations and found out that the higher-order theory results are more accurate than lower-order ones for some vibration modes. Therefore, more solutions of vibration analysis prefer higher-order plate theory and finite element methods regarding design tool for development. However, using such solutions usually means solving a very large matrix problem in order to obtain the eigenvalues as vibration frequencies. In the traditional linear algebra problems, this means a lot of computer memory and computing time which greatly affect the computational efficiency. Lately, Wang, et al. ${ }^{15}$ have developed a finite element program with parallel computing capabilities on Linux clusters to quickly handle sparse matrices and algebraic operations with software components from public domains and this effectively improved the computational efficiency of high frequency vibration problems. Subsequently, they also implemented nonlinear finite element analysis with the program. 16,17

Currently, the Mindlin plate equations with truncation and corrections for parallel finite element analysis are up to the fifth-order or even higher which also takes into account the potential and overtone displacement variations. ${ }^{18}$ At the same time, the analysis of nonlinear vibrations is also included. ${ }^{16,17,19}$ Generally speaking, with the higher-order plate theory, considerations of nonlinearity, and other factors to improve the accuracy and parallel computing technology to improve the computation efficiency, we can effectively obtain the vibration solutions with great accuracy. For the design of the quartz crystal resonators, we still need the exact mode from the massive calculation results by plotting the mode shapes. There is a lack of efficiency because it requires manual interventions which will affect the automation of the parallel computing.

Based on the fact that the vibration energies are stored in each vibration mode, the energy method can be used for vibration analysis, as shown in modal strain energy approach..$^{20}$ For piezoelectric plate vibrations, the energy calculations were often used to describe the energy trapping and harvest effect. ${ }^{21,22}$ Through the research of Yong, et al., ${ }^{23}$ the ratios of the trapped energies can be calculated to evaluate the quality of resonators.

Previously, the use of energy methods in vibrations has been common in structural dynamics and the calculated energy has been used for the identification of vibration modes in a complex structure. ${ }^{24}$ The statistical energy analysis (SEA) is a mature method for the efficient calculation of the response of structural vibration. ${ }^{25}$ In a different approach from the traditional solutions of mechanical vibrations, the statistical method uses the energy of each subsystem as the basic parameters to solve the vibration problem. ${ }^{26}$

Besides SEA, there are also other methods which utilize the energy contributions of vibration modes to make more accurate approximations to vibrations ${ }^{27}$ such as the detection of dominant modes of shell structures under external excitations like wind or ground motion ${ }^{28,29}$ and using dominating energy distribution to characterize the vibration signals. ${ }^{30}$ In this paper, we focus on the high frequency vibration analysis of the quartz crystal resonator and target locating the pure thickness-shear mode from the coupled vibrations with energy formulation at each frequency with multiple vibration modes or degree-offreedoms (DOFs).

To serve the purpose of identifying the dominant mode of a quartz crystal resonator, the strain and kinetic energies of vibrations with multiple DOFs are presented with formulations by vibration solutions at each frequency. Using the strain energy of each DOF to distinguish the dominant mode of a gear pair determined effective as described by Wang, et al. ${ }^{31}$ Unlike using the time-averaged kinetic energy, ${ }^{28-30}$ in this research, the strain and kinetic energies in each vibration cycle are calculated because the quartz crystal resonator exhibits perfectly steady vibrations. As a result, both the kinetic and strain energy distributions in a vibration cycle are used to identify the dominant functioning mode with the highest energy concentration.

To demonstrate the use of the energy-based vibration mode identification method, we chose the well-known Mindlin firstorder equations of a rectangular plate for calculations. First, we adopted the first-order plate equations and calculated the displacement expressions of vibration modes. Then, we established the theoretical formulation of the kinetic and strain energy densities and integrated them over the plate for total energy. Next, we divided the total energy according to specific mode types and calculated the energy distributions of each vibration mode. We calculated the energies in three different material types, which were two different sets of mode couplings with an AT-cut and a full set of mode couplings with an SC-cut quartz crystal plate. Finally, we successfully observed the functioning thickness-shear mode from the highest concen- 


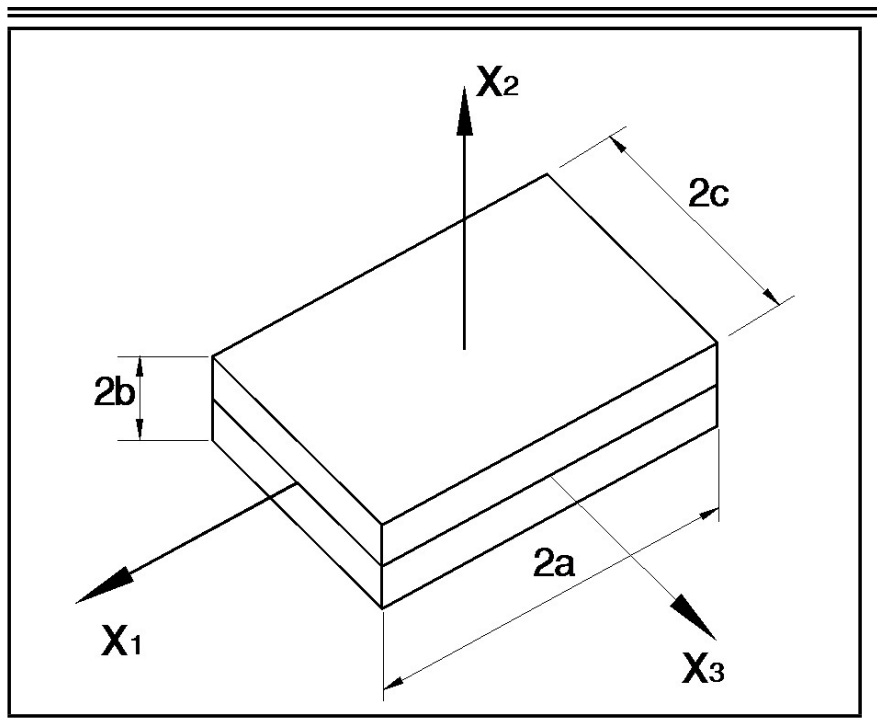

Figure 1. The simple model of a plate for the crystal resonator.

trations of kinetic and strain energies. The results were also confirmed by mode shapes at each resonant frequency by the traditional mode plotting method. ${ }^{32}$ This energy approach can be effective and easy to use in the vibration mode identification and is capable of improving the features of finite element analysis.

\section{THE FIRST-ORDER EQUATIONS OF THE MINDLIN PLATE THEORY}

For the analysis of thickness-shear vibrations of a quartz crystal plate, the first-order Mindlin plate equations with limited couplings of modes are adequate for accurate results. ${ }^{1-5}$ We chose a rectangular plate with large length to thickness ratio for the calculations, and the analytical solutions based on the straight-crested wave assumptions are useful in the design and optimization of quartz crystal resonators.

As known in fundamental thickness-shear vibrations of quartz crystal resonators, there are six modes to be analyzed based on the Mindlin first-order plate theory, which are the extension (E) $u_{1}^{(0)}$, flexural (F) $u_{2}^{(0)}$, face-shear (FS) $u_{3}^{(0)}$, thickness-shear (TSh) $u_{1}^{(1)}$, thickness-stretch (TSt) $u_{2}^{(1)}$, and thickness-twist (TT) $u_{3}^{(1)} \cdot 8$ However, in different material types the coupling modes are different with the number of modes in the solutions. The simple model of a plate for the crystal resonator we used here is a rectangular AT-cut quartz crystal plate with four free sides as shown in Fig. 1 with $2 b$, $2 a$ and $2 c$ representing its thickness, length, and width, respectively.

To demonstrate our energy approach, we utilized the equations without electrodes, ${ }^{10,33,34}$ without external forces, ${ }^{35}$ and with temperature at $25^{\circ} \mathrm{C}$ to avoid frequency-temperature effect. ${ }^{36,37}$ Furthermore, only the straight-crested waves traveling along the $x_{1}$ direction are considered, making the equations greatly simplified.

With the given plate model and boundary conditions in Fig. 1, we have the stress-displacement equations for quartz crystal plate with the full set of elastic constants and modifica- tions as follows ${ }^{8,38}$

$$
\begin{aligned}
T_{j}^{(0)}= & 2 b \kappa_{j}^{(0)}\left[c_{j 1} u_{1,1}^{(0)}+c_{j 2} \kappa_{2}^{(0)} u_{2}^{(1)}+c_{j 3} u_{3,3}^{(0)}\right. \\
& +c_{j 4} \kappa_{4}^{(0)}\left(u_{2,3}^{(0)}+u_{3}^{(1)}\right)+c_{j 5}\left(u_{3,1}^{(0)}+u_{1,3}^{(0)}\right) \\
& \left.+c_{j 6} \kappa_{6}^{(0)}\left(u_{2,1}^{(0)}+u_{1}^{(1)}\right)\right] ; \\
T_{j}^{(1)}= & \frac{2 b^{3}}{3} \kappa_{j}^{(1)}\left[\bar{c}_{j 1} u_{1,1}^{(1)}+\bar{c}_{13} u_{3,3}^{(1)}+\bar{c}_{14} \kappa_{4}^{(1)} u_{2,3}^{(1)}\right. \\
& \left.+\bar{c}_{15}\left(u_{3,1}^{(1)}+\bar{c}_{15} u_{1,3}^{(1)}\right)+\bar{c}_{16} \kappa_{6}^{(1)} u_{2,1}^{(1)}\right] ; \\
T_{2}^{(1)}= & 0, \bar{c}_{\mathrm{ij}}=c_{\mathrm{ij}}-\frac{c_{i 2} c_{2 j}}{c_{22}}, i, j=1,2,3,4,5,6 ;
\end{aligned}
$$

where $T_{j}^{(n)}(n=0,1 ; j=1,2, \ldots 6)$ are the $n$ th-order stresses, and $c_{\mathrm{ij}}$ are the elastic constants of quartz crystal. To correct the discrepancy due to the truncation of higher-order flexural mode, elastic constants $c_{\mathrm{ij}}$ have been modified. ${ }^{1}$ The constant $\kappa_{j}^{(n)}$ are the correction factors to compensate the truncation and their value are $2,6,8,38-40$

$$
\begin{gathered}
\kappa_{p}^{(0)}=\frac{\pi}{2 \sqrt{3}}, \kappa_{p}^{(1)}=1, p=2,4,6 ; \\
\kappa_{q}^{(0)}=1, \kappa_{q}^{(1)}=1, q=1,3,5 .
\end{gathered}
$$

The corresponding two-dimensional equations of motion of the six vibration modes are ${ }^{8}$

$$
\begin{gathered}
\frac{\partial T_{1}^{(0)}}{\partial x_{1}}+\frac{\partial T_{5}^{(0)}}{\partial x_{3}}=2 b \rho \ddot{u}_{1}^{(0)} ; \\
\frac{\partial T_{6}^{(0)}}{\partial x_{1}}+\frac{\partial T_{4}^{(0)}}{\partial x_{3}}=2 b \rho \ddot{u}_{2}^{(0)} ; \\
\frac{\partial T_{5}^{(0)}}{\partial x_{1}}+\frac{\partial T_{3}^{(0)}}{\partial x_{3}}=2 b \rho \ddot{u}_{3}^{(0)} ; \\
\frac{\partial T_{1}^{(1)}}{\partial x_{1}}+\frac{\partial T_{5}^{(1)}}{\partial x_{3}}-T_{6}^{(0)}=\frac{2 b^{3}}{3} \rho \ddot{u}_{1}^{(1)} ; \\
\frac{\partial T_{6}^{(1)}}{\partial x_{1}}+\frac{\partial T_{4}^{(1)}}{\partial x_{3}}-T_{2}^{(0)}=\frac{2 b^{3}}{3} \rho \ddot{u}_{2}^{(1)} ; \\
\frac{\partial T_{5}^{(1)}}{\partial x_{1}}+\frac{\partial T_{3}^{(1)}}{\partial x_{3}}-T_{4}^{(0)}=\frac{2 b^{3}}{3} \rho \ddot{u}_{3}^{(1)} ;
\end{gathered}
$$

where $\rho$ is the density of quartz crystal.

The six displacements of straight-crested waves travelling along the $x_{1}$ direction are defined as ${ }^{1,8}$

$$
u_{j}^{(0)}=A_{j} \sin \xi x_{1} e^{i \omega t}
$$

$$
u_{j}^{(1)}=\frac{A_{j+3}}{b} \cos \xi x_{1} e^{i \omega t}, j=1,2,3 ;
$$




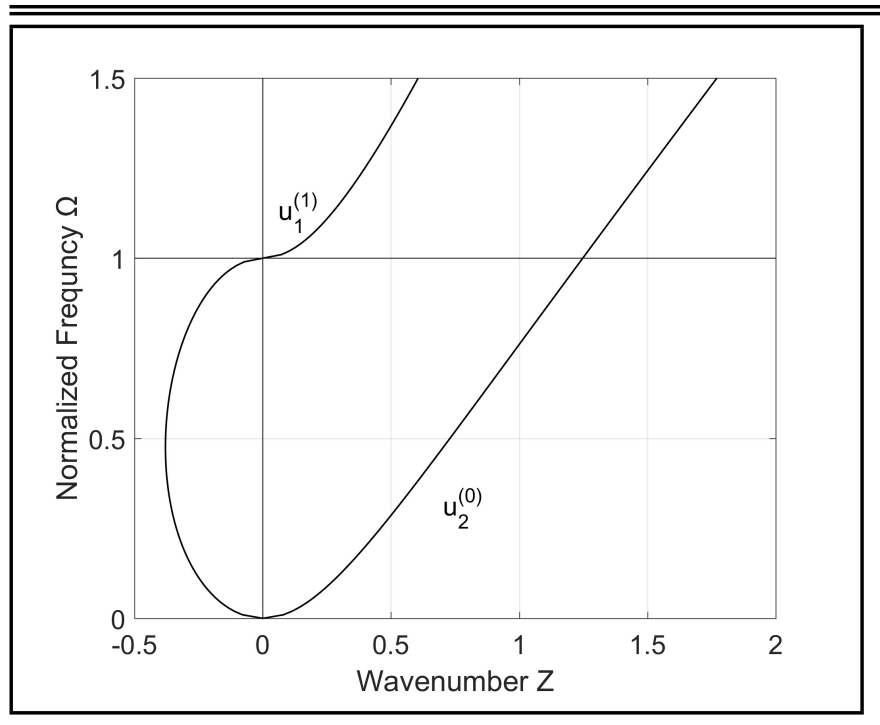

Figure 2. Dispersion relations of coupled thickness-shear and flexural vibrations of an AT-cut quartz crystal plate.

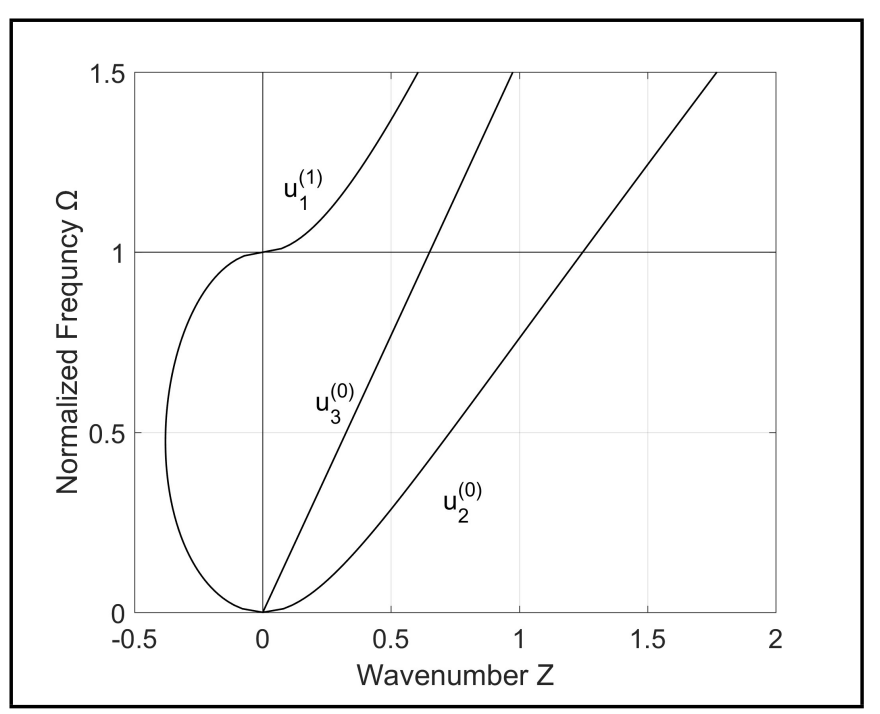

Figure 3. Dispersion relations of coupled thickness-shear, flexural, and faceshear vibrations of an AT-cut quartz crystal plate.

where $A_{i}(i=1,2,3,4,5,6), \xi, \omega$ are the amplitudes, wavenumber, and vibration frequency, respectively.

In this paper, there are three mode coupling combinations as shown below, ${ }^{3,14}$

$\begin{cases}u_{2}^{(0)}, u_{1}^{(1)}, & \text { AT - cut, two modes; } \\ u_{2}^{(0)}, u_{3}^{(0)}, u_{1}^{(1)}, & \text { AT - cut, three modes; } \\ u_{1}^{(0)}, u_{2}^{(0)}, u_{3}^{(0)}, u_{1}^{(1)}, u_{2}^{(1)}, u_{3}^{(1)}, & \text { SC - cut, six modes. }\end{cases}$

By substituting Eq. (5) according to Eq. (4) into Eqs. (1) and (3), we can obtain the dispersion relations as functions of the normalized frequency $\Omega$ and wavenumber $Z$ as

$$
\mathbf{M}(\Omega, Z)=0
$$

where

$$
\Omega=\frac{\omega}{\omega_{0}}, \omega_{0}=\frac{\pi}{2 b} \sqrt{\frac{c_{66}}{\rho}}, Z=\frac{\xi}{\frac{\pi}{2 b}},
$$

and $\mathbf{M}$ stands for the dispersion relation matrix of different mode types and we give the detail later in the Appendix.

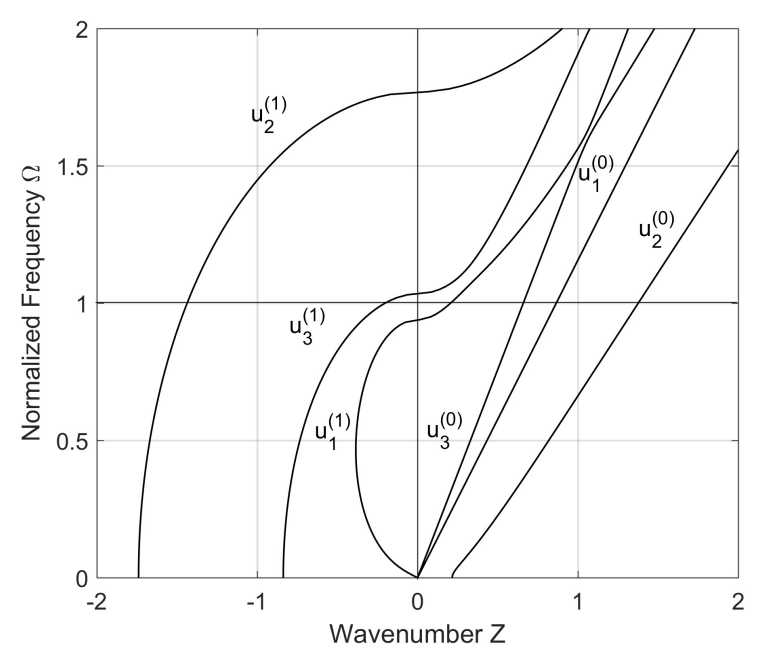

Figure 4. Dispersion relations of coupled thickness-shear, thickness-stretch, thickness-twist, extension, flexural, and face-shear vibrations of an SC-cut quartz crystal plate.

By solving Eq. (6), we can obtain the dispersion relations of a quartz crystal plate as shown in Figs. 2-4. The dispersion relations validated the equations and also revealed the characteristics of waves in the plate.

With the above results of normalized wavenumbers at each frequency, we can rewrite the displacements of Eq. (4) as

$$
\begin{array}{r}
u_{j}^{(0)}=\sum_{r=1}^{6}\left[\alpha_{j r} \beta_{r} A_{44} \sin \left(\frac{\pi Z_{r}}{2} \frac{x_{1}}{b}\right)\right] e^{i \omega t} ; \\
u_{j}^{(1)}=\sum_{r=1}^{6}\left[\alpha_{(j+3) r} \beta_{r} \frac{A_{44}}{b} \cos \left(\frac{\pi Z_{r}}{2} \frac{x_{1}}{b}\right)\right] e^{i \omega t}, j=1,2,3 ;
\end{array}
$$

where $Z_{r}(r=1,2,3,4,5,6)$ are the the normalized wavenumbers, $A_{44}$ stands for the amplitude $A_{4}$ at the fourth wavenumber and $\alpha_{j r}$ are the corresponding amplitude ratios defined as:

$\alpha_{j r}=\frac{A_{j r}}{A_{4 r}}, j, r= \begin{cases}2,4, & \text { AT - cut, two modes; } \\ 2,3,4, & \text { AT - cut, three modes; } \\ 1,2,3,4,5,6, & \text { SC - cut, six modes }\end{cases}$

$$
\beta_{r}=\frac{A_{4 r}}{A_{44}}, r= \begin{cases}2,4, & \text { AT - cut }, \text { two modes } \\ 2,3,4, & \text { AT - cut three modes } \\ 1,2,3,4,5,6, & \text { SC - cut }, \text { six modes }\end{cases}
$$

where $A_{j r}$ is the amplitude $A_{j}$ at the $r$ th wavenumber.

Using the traction-free boundary conditions of the plate shown in Fig. 1, the corresponding stress boundary conditions at $x_{1}= \pm a$ are:

$$
T_{5}^{(0)}=T_{6}^{(0)}=T_{1}^{(0)}=T_{5}^{(1)}=T_{6}^{(1)}=T_{1}^{(1)}=0 .
$$

By substituting Eq. (7) to Eq. (1) then to Eq. (9), we can solve the frequency equations

$$
\left|\mathbf{N}\left(\beta_{r}, Z_{r}\right)\right|=0 ;
$$


where $\mathbf{N}$ stands for the coefficient matrix of frequency equation of the coupled modes and we give the details in the Appendix.

Let the coefficient determinant of Eq. (10) vanishing, we can obtain the frequency spectra as frequency versus length to thickness ratio. Then, from the dispersion relations, we can get a set of wavenumbers $Z_{r}$ at a specific frequency $\Omega$. By substituting $Z_{r}$ back into Eq. (10), we then obtain $\beta_{r}$.

With displacements solved in Eq. (7) and for the calculation of strain energies later, we also give the expressions of nontrivial strains with displacements of the $x_{1}$-propagating straightcrested waves as:

$$
\begin{aligned}
& S_{1}^{(0)}=u_{1,1}^{(0)} ; \quad S_{4}^{(0)}=u_{3}^{(1)} ; \quad S_{2}^{(0)}=u_{2}^{(1)} \\
& S_{5}^{(0)}=u_{3,1}^{(0)} ; \quad S_{3}^{(0)}=0 ; \quad S_{6}^{(0)}=u_{2,1}^{(0)}+u_{1}^{(1)} ; \\
& S_{1}^{(1)}=u_{1,1}^{(1)} ; \quad S_{4}^{(1)}=0 ; \quad S_{2}^{(1)}=2 u_{2}^{(2)} \\
& S_{5}^{(1)}=u_{3,1}^{(1)} ; \quad S_{3}^{(1)}=0 ; \quad S_{6}^{(1)}=u_{2,1}^{(1)} .
\end{aligned}
$$

All the derivation above is primarily based on the first-order Mindlin plate theory. ${ }^{3,8}$ After all the displacements and strains in Eqs. (7) and (8) are obtained, the calculation of the strain and kinetic energies of the plate can be carried out without any difficulty.

To obtain the dominant vibration modes, it needs the vibration mode shapes from Eq. (7) to find out the largest amplitude for each frequency in the frequency spectra. With this process, all the curves in the spectra will be labeled by the dominant vibration mode and the optimal selection of length, or the length to thickness ratio, can also be made from frequency spectra. Clearly, it is a lengthy and tedious procedure requiring the calculation and plotting of vibration modes with a visual check by an experienced researcher. In case of numerical analysis with a larger set of data and solutions, the plotting of vibration modes is the last choice of the vibration mode identification procedure we can consider.

\section{THE CALCULATION OF ENERGIES OF VIBRATIONS OF A PLATE}

To enable a formulation and calculation with displacement solutions for the vibration mode identification, it is natural to turn to the energy of vibrations for a possible solution. The idea is that the largest vibration mode should be the one with the largest proportion of the strain energy or kinetic energy. Then the identification of vibration modes can be made by checking the energy proportion from vibration solutions. Clearly, it is simpler to calculate and compare the energy proportion in both analytical and numerical solutions for this purpose. To this objective, we first need to obtain the energy formulation with vibration solutions.

\subsection{The Strain Energy}

First, the strain energy is the energy stored in the plate when it undergoes deformation in vibrations. By analyzing strain energy of the plate, the relations of stresses, strains, and displacements will be merged, implying a simple result for the evaluation of the state of vibrations. We start with the strain energy density which can be used to represent the energy in the plate through integration. With the first-order Mindlin plate theory of selected modes, the strain energy density is ${ }^{8}$

$$
\begin{aligned}
\bar{U} & =\frac{1}{2} c_{p q} \sum_{m} \sum_{n} B_{m n} S_{p}^{(m)} S_{q}^{(n)} \\
& =\frac{1}{2} c_{p q}\left(B_{00} S_{p}^{(0)} S_{q}^{(0)}+B_{11} S_{p}^{(1)} S_{q}^{(1)}\right)
\end{aligned}
$$

where $B_{m n}(m, n=0,1)$ and $S_{p}^{(n)}(p=1,2,3,4,5,6 ; n=$ $0,1)$ are the integral constants and strains of the $n$ th-order as given in Eq. (12), respectively. The energy varies with different cases of mode coupling as we show in the following.

\subsubsection{AT-cut quartz crystal plates with two vibration modes}

As the case of two modes $u_{2}^{(0)}$ and $u_{1}^{(1)}$, Eq. (11) is simplified with zero displacements and the Eq. (12) becomes:

$$
\begin{aligned}
\bar{U}= & \frac{1}{2} c_{66} B_{00} S_{6}^{(0)} S_{6}^{(0)}+\frac{1}{2} c_{11} B_{11} S_{1}^{(1)} S_{1}^{(1)} \\
& +\frac{1}{2} c_{22} B_{11} S_{2}^{(1)} S_{2}^{(1)}+c_{12} B_{11} S_{1}^{(1)} S_{2}^{(1)} \\
= & \frac{1}{2} B_{00} c_{66} \cdot\left(u_{2,1}^{(0)}\right)^{2}+\frac{1}{2} B_{00} c_{66} \cdot\left(u_{1}^{(1)}\right)^{2} \\
& +\frac{1}{2} B_{11} \bar{c}_{11} \cdot\left(u_{1,1}^{(1)}\right)^{2}+B_{00} c_{66} \cdot u_{2,1}^{(0)} u_{1}^{(1)} .
\end{aligned}
$$

Next, we classify the energy densities according to the vibration modes in Eq. (13) as follows:

$$
\begin{gathered}
\bar{U}_{\mathrm{TSh}}=\frac{1}{2} B_{00} c_{66}\left(u_{1}^{(1)}\right)^{2}+\frac{1}{2} B_{11} \bar{c}_{11}\left(u_{1,1}^{(1)}\right)^{2} ; \\
\bar{U}_{\mathrm{F}}=\frac{1}{2} B_{00} c_{66}\left(u_{2,1}^{(0)}\right)^{2} ; \\
\bar{U}_{\mathrm{F}-\mathrm{TSh}}=B_{00} c_{66} u_{2,1}^{(0)} u_{1}^{(1)}
\end{gathered}
$$

where $\bar{U}_{\mathrm{TSh}}$ and $\bar{U}_{\mathrm{F}}$ are strain energy densities of single thickness-shear and flexural mode, while $\bar{U}_{\mathrm{F}-\mathrm{TSh}}$ is of the coupled ones.

Finally, the total strain energies of the quartz crystal plate are obtained by integrating the energy densities of Eq. (14) in 
the entire plate in one vibration cycle as:

$$
U_{\mathrm{X}}=\int_{-a}^{a} \int_{-c}^{c} \int_{0}^{T} \bar{U}_{X} \mathrm{~d} x_{1} \mathrm{~d} x_{3} \mathrm{~d} t, \mathrm{X}=\mathrm{TSh}, \mathrm{F}, \mathrm{F}-\mathrm{TSh} ;
$$

$$
\begin{aligned}
U_{\mathrm{TSh}}= & \frac{2 \pi c}{\omega} c_{66}\left\{\sum_{i=1}^{2} A_{4 i}^{2} \alpha_{4 i}^{2} V_{c 2}+\sum_{i=1, j \neq i}^{2} A_{4 i} A_{4 j} \alpha_{4 i} \alpha_{4 j} V_{\mathrm{cc}}\right\} \\
& +\frac{\pi^{3} c}{6 \omega} \bar{c}_{11}\left\{\sum_{i=1}^{2} A_{4 i}^{2} \alpha_{4 i}^{2} Z_{i}^{2} V_{s 2}\right. \\
& \left.+\sum_{i=1, j \neq i}^{2} A_{4 i} A_{4 j} \alpha_{4 i} \alpha_{4 j} Z_{i} Z_{j} V_{\mathrm{ss}}\right\} \\
U_{\mathrm{F}}= & \frac{\pi^{3} c}{\omega} c_{66}\left\{\sum_{i=1}^{2} A_{4 i}^{2} \alpha_{2 i}^{2} Z_{i}^{2} V_{c 2}\right. \\
& \left.+\sum_{i=1, j \neq i}^{2} A_{4 i} A_{4 j} \alpha_{2 i} \alpha_{2 j} Z_{i} Z_{j} V_{\mathrm{cc}}\right\}
\end{aligned}
$$

$$
\begin{aligned}
U_{\mathrm{F}-\mathrm{TSh}}= & \frac{4 \pi^{3} c}{\omega} c_{66}\left\{\sum_{i=1}^{2} A_{4 i} A_{4 i} \alpha_{2 i} \alpha_{4 i} Z_{i} V_{c 2}\right. \\
& \left.+\sum_{i=1, j \neq i}^{2} A_{4 i} A_{4 j} \alpha_{2 i} \alpha_{4 j} Z_{i} V_{\mathrm{cc}}\right\} ;
\end{aligned}
$$

$U=\int_{-a}^{a} \int_{-c}^{c} \int_{0}^{T} \bar{U} \mathrm{~d} x_{1} \mathrm{~d} x_{3} \mathrm{~d} t=U_{\mathrm{TSh}}+U_{\mathrm{F}}+U_{\mathrm{F}-\mathrm{TSh}}, T=\frac{2 \pi}{\omega} ;$

where $T$ is the period of vibration, $U$ is the total strain energy, $U_{\mathrm{TSh}}$ and $U_{\mathrm{F}}$ are the strain energy of thickness-shear and flexural modes, and $U_{\mathrm{F}-\mathrm{TSh}}$ is the coupled energy, respectively. And the coefficients $V_{c 2}, V_{\mathrm{cc}}, V_{s 2}$, and $V_{\mathrm{ss}}$ in Eq. (15) are given as:

$$
\begin{aligned}
V_{c 2} & =\frac{1}{b} \int_{-a}^{a} \cos ^{2}\left(\frac{\pi Z_{i}}{2} \frac{x_{1}}{b}\right) \mathrm{d} x_{1}=\frac{a}{b}+\frac{1}{\pi Z_{i}} \sin \left(\frac{a}{b} \pi Z_{i}\right) ; \\
V_{\mathrm{cc}} & =\frac{1}{b} \int_{-a}^{a} \cos \left(\frac{\pi Z_{i}}{2} \frac{x_{1}}{b}\right) \cos \left(\frac{\pi Z_{j}}{2} \frac{x_{1}}{b}\right) \mathrm{d} x_{1} \\
& =\frac{2}{\pi}\left[\frac{\sin \left(\frac{a}{b} \frac{\pi}{2}\left(Z_{i}-Z_{j}\right)\right)}{Z_{i}-Z_{j}}+\frac{\sin \left(\frac{a}{b} \frac{\pi}{2}\left(Z_{i}+Z_{j}\right)\right)}{Z_{i}+Z_{j}}\right] ;
\end{aligned}
$$

$$
V_{s 2}=\frac{1}{b} \int_{-a}^{a} \sin ^{2}\left(\frac{\pi Z_{i}}{2} \frac{x_{1}}{b}\right) \mathrm{d} x_{1}=\frac{a}{b}-\frac{1}{\pi Z_{i}} \sin \left(\frac{a}{b} \pi Z_{i}\right) ;
$$$$
V_{\mathrm{ss}}=\frac{1}{b} \int_{-a}^{a} \sin \left(\frac{\pi Z_{i}}{2} \frac{x_{1}}{b}\right) \sin \left(\frac{\pi Z_{j}}{2} \frac{x_{1}}{b}\right) \mathrm{d} x_{1}
$$$$
=\frac{2}{\pi}\left[\frac{\sin \left(\frac{a}{b} \frac{\pi}{2}\left(Z_{i}-Z_{j}\right)\right)}{Z_{i}-Z_{j}}-\frac{\sin \left(\frac{a}{b} \frac{\pi}{2}\left(Z_{i}+Z_{j}\right)\right)}{Z_{i}+Z_{j}}\right] \text {. }
$$

\subsubsection{AT-cut quartz crystal plate with three vibration modes}

With the same procedure, we can obtain the strain energy density of the case of three modes as:

$$
\begin{aligned}
\bar{U}= & \frac{1}{2} c_{55} B_{00} S_{5}^{(0)} S_{5}^{(0)}+\frac{1}{2} c_{66} B_{00} S_{6}^{(0)} S_{6}^{(0)}+c_{56} B_{00} S_{5}^{(0)} S_{6}^{(0)} \\
& +\frac{1}{2} c_{11} B_{11} S_{1}^{(1)} S_{1}^{(1)}+\frac{1}{2} c_{22} B_{11} S_{2}^{(1)} S_{2}^{(1)}+c_{12} B_{11} S_{1}^{(1)} S_{2}^{(1)} \\
= & \frac{1}{2} B_{00} c_{66} \cdot\left(u_{2,1}^{(0)}\right)^{2}+\frac{1}{2} B_{00} c_{55} \cdot\left(u_{3,1}^{(0)}\right)^{2}+\frac{1}{2} B_{00} c_{66} \cdot\left(u_{1}^{(1)}\right)^{2} \\
& +\frac{1}{2} B_{11} \bar{c}_{11} \cdot\left(u_{1,1}^{(1)}\right)^{2}+B_{00} c_{66} \cdot u_{2,1}^{(0)} u_{1}^{(1)} \\
& +B_{00} c_{65} \cdot u_{2,1}^{(0)} u_{3,1}^{(0)}+B_{00} c_{65} \cdot u_{1}^{(1)} u_{3,1}^{(0)}
\end{aligned}
$$

Next, we classify the energy densities according to the vibration modes in Eq. (17) as follows:

$$
\begin{aligned}
\bar{U}_{\mathrm{TSh}} & =\frac{1}{2} B_{00} c_{66}\left(u_{1}^{(1)}\right)^{2}+\frac{1}{2} B_{11} \bar{c}_{11}\left(u_{1,1}^{(1)}\right)^{2} ; \\
\bar{U}_{\mathrm{F}} & =\frac{1}{2} B_{00} c_{66}\left(u_{2,1}^{(0)}\right)^{2} ; \\
\bar{U}_{\mathrm{FS}} & =\frac{1}{2} B_{00} c_{55}\left(u_{3,1}^{(0)}\right)^{2} ; \\
\bar{U}_{\mathrm{F}-\mathrm{TSh}} & =B_{00} c_{66} u_{2,1}^{(0)} u_{1}^{(1)} ; \\
\bar{U}_{\mathrm{F}-\mathrm{FS}} & =B_{00} c_{65} u_{2,1}^{(0)} u_{3,1}^{(0)} ; \\
\bar{U}_{\mathrm{TSh}-\mathrm{FS}} & =B_{00} c_{65} u_{1}^{(1)} u_{3,1}^{(0)} ;
\end{aligned}
$$

where $\bar{U}_{\mathrm{X}}(\mathrm{X}=\mathrm{TSh}, \mathrm{F}, \mathrm{FS}, \mathrm{F}-\mathrm{TSh}, \mathrm{F}$ - FS, TSh - FS) are the strain energies of different modes. Since for AT-cut quartz crystal $c_{65}$ is much smaller, it is clear that the coupled energies should be much smaller in comparison with the energies of single modes. Thus, these coupled energies are neglected in following calculations.

Finally, the total strain energies of the quartz crystal plate are obtained by integrating the energy densities of Eq. (18) in the entire plate in one vibration cycle as:

$$
U_{\mathrm{X}}=\int_{-a}^{a} \int_{-c}^{c} \int_{0}^{T} \bar{U}_{X} \mathrm{~d} x_{1} \mathrm{~d} x_{3} \mathrm{~d} t
$$

$\mathrm{X}=$ TSh, F, FS, F - TSh, F - FS, TSh - FS; (19a)

$$
\begin{aligned}
U_{\mathrm{TSh}}= & \frac{2 \pi c}{\omega} c_{66}\left\{\sum_{i=1}^{3} A_{4 i}^{2} \alpha_{4 i}^{2} V_{c 2}\right. \\
& \left.+\sum_{i=1, j \neq i}^{3} A_{4 i} A_{4 j} \alpha_{4 i} \alpha_{4 j} V_{\mathrm{cc}}\right\} \\
& +\frac{\pi^{3} c}{6 \omega} \bar{c}_{11}\left\{\sum_{i=1}^{3} A_{4 i}^{2} \alpha_{4 i}^{2} Z_{i}^{2} V_{s 2}\right.
\end{aligned}
$$




$$
\begin{aligned}
& \left.+\sum_{i=1, j \neq i}^{3} A_{4 i} A_{4 j} \alpha_{4 i} \alpha_{4 j} Z_{i} Z_{j} V_{\mathrm{ss}}\right\} \\
& U_{\mathrm{F}}=\frac{\pi^{3} c}{\omega} c_{66}\left\{\sum_{i=1}^{3} A_{4 i}^{2} \alpha_{2 i}^{2} Z_{i}^{2} V_{c 2}\right. \\
& \left.+\sum_{i=1, j \neq i}^{3} A_{4 i} A_{4 j} \alpha_{2 i} \alpha_{2 j} Z_{i} Z_{j} V_{\mathrm{cc}}\right\} \text {; } \\
& U_{\mathrm{FS}}=\frac{\pi^{3} c}{\omega} c_{55}\left\{\sum_{i=1}^{3} A_{4 i}^{2} \alpha_{3 i}^{2} Z_{i}^{2} V_{c 2}\right. \\
& \left.+\sum_{i=1, j \neq i}^{3} A_{4 i} A_{4 j} \alpha_{3 i} \alpha_{3 j} Z_{i} Z_{j} V_{\mathrm{cc}}\right\} \\
& U_{\mathrm{F}-\mathrm{TSh}}=\frac{4 \pi^{3} c}{\omega} c_{66}\left\{\sum_{i=2}^{4} A_{4 i} A_{4 i} \alpha_{2 i} \alpha_{4 i} Z_{i} V_{c 2}\right. \\
& \left.+\sum_{i=2, j \neq i}^{4} A_{4 i} A_{4 j} \alpha_{2 i} \alpha_{4 j} Z_{i} V_{\mathrm{cc}}\right\} \\
& +\frac{1}{2} \sum_{p=1,2,5,6} \sum_{q=1,2,5,6} c_{\mathrm{pq}} B_{11} S_{p}^{(1)} S_{q}^{(1)} \\
& =\frac{1}{2} B_{00}\left[c_{11}\left(u_{1,1}^{(0)}\right)^{2}+c_{22}\left(u_{2}^{(1)}\right)^{2}+c_{44}\left(u_{3}^{(1)}\right)^{2}\right. \\
& \left.+c_{55}\left(u_{3,1}^{(0)}\right)^{2}+c_{66}\left(u_{2,1}^{(0)}\right)^{2}+c_{66}\left(u_{1}^{(1)}\right)^{2}\right] \\
& +B_{00}\left[c_{66} u_{2,1}^{(0)} u_{1}^{(1)}+c_{12} u_{1,1}^{(0)} u_{2}^{(1)}+c_{14} u_{1,1}^{(0)} u_{3}^{(1)}\right. \\
& \left.+c_{15} u_{1,1}^{(0)} u_{3,1}^{(0)}+c_{16} u_{1,1}^{(0)}\left(u_{2,1}^{(0)}+u_{1}^{(1)}\right)+c_{24} u_{2}^{(1)} u_{3}^{(1)}\right] \\
& +B_{00}\left[c_{25} u_{2}^{(1)} u_{3,1}^{(0)}+c_{26} u_{2}^{(1)}\left(u_{2,1}^{(0)}+u_{1}^{(1)}\right)+c_{45} u_{3}^{(1)} u_{3,1}^{(0)}\right. \\
& \left.+c_{46} u_{3}^{(1)}\left(u_{2,1}^{(0)}+u_{1}^{(1)}\right)+c_{56} u_{3,1}^{(0)}\left(u_{2,1}^{(0)}+u_{1}^{(1)}\right)\right] \\
& +\frac{1}{2} B_{11}\left[\bar{c}_{11}\left(u_{1,1}^{(1)}\right)^{2}+\bar{c}_{55}\left(u_{3,1}^{(1)}\right)^{2}+\bar{c}_{66}\left(u_{2,1}^{(1)}\right)^{2}\right] \\
& +B_{11}\left[\bar{c}_{16} u_{2,1}^{(1)} u_{1}^{(1)}+\bar{c}_{15} u_{1,1}^{(1)} u_{3,1}^{(1)}+\bar{c}_{65} u_{2,1}^{(1)} u_{3,1}^{(1)}\right] \text {. }
\end{aligned}
$$$$
U_{\mathrm{F}-\mathrm{FS}}=\frac{2 \pi^{4} c}{\omega} c_{65}\left\{\sum_{i=1}^{3} A_{4 i} A_{4 i} \alpha_{3 i} \alpha_{2 i} Z_{i}^{2} V_{c 2}\right.
$$$$
\left.+\sum_{i=1, j \neq i}^{3} A_{4 i} A_{4 j} \alpha_{3 i} \alpha_{2 j} Z_{i}^{2} V_{\mathrm{cc}}\right\} \text {; }
$$$$
U_{\text {TSh - FS }}=\frac{4 \pi^{3} c}{\omega} c_{65}\left\{\sum_{i=1}^{3} A_{4 i} A_{4 i} \alpha_{4 i} \alpha_{3 i} Z_{i} V_{c 2}\right.
$$$$
\left.+\sum_{i=1, j \neq i}^{3} A_{4 i} A_{4 j} \alpha_{4 i} \alpha_{3 j} Z_{i} V_{\mathrm{cc}}\right\}
$$$$
U=\int_{-a}^{a} \int_{-c}^{c} \int_{0}^{T} \bar{U} \mathrm{~d} x_{1} \mathrm{~d} x_{3} \mathrm{~d} t
$$$$
=U_{\mathrm{TSh}}+U_{\mathrm{F}}+U_{\mathrm{FS}}+U_{\mathrm{F}-\mathrm{TSh}}+U_{\mathrm{F}-\mathrm{FS}}+U_{\mathrm{TSh}-\mathrm{FS}}
$$

where $U$ is the total strain energy, $U_{\mathrm{TSh}}, U_{\mathrm{F}}$, and $U_{\mathrm{FS}}$ are the strain energy of thickness-shear, flexural and face-shear modes, and $U_{\mathrm{F}-\mathrm{TSh}}, U_{\mathrm{F}-\mathrm{FS}}$, and $U_{\mathrm{TSh}-\mathrm{FS}}$ are the coupled energies, respectively.

\subsubsection{SC-cut quartz crystal plates with six vibration modes}

The SC-cut quartz crystal is one of the most complicated material types in vibration analysis with the first-order Mindlin plate equations as the elastic constant matrix is full. Due to the material characteristics of this special orientation, all the six vibration modes are coupled and need to be calculated in the energy solutions. First, we can obtain the strain energy density of six modes as:

$\overline{\bar{U}=\frac{1}{2} \sum_{p=1,2,4,5,6} \sum_{q=1,2,4,5,6} c_{\mathrm{pq}} B_{00} S_{p}^{(0)} S_{q}^{(0)}}$

Observing the Eq. (20), though there are many terms of coupled energy, but many elastic constants associated with them are smaller in comparison to the single mode terms. As a result, the coupled energies are also neglected here, and only the larger energy density terms are presented as:

$$
\begin{aligned}
\bar{U}_{\mathrm{TSh}} & =\frac{1}{2} B_{00} c_{66}\left(u_{1}^{(1)}\right)^{2}+\frac{1}{2} B_{11} \bar{c}_{11}\left(u_{1,1}^{(1)}\right)^{2} ; \\
\bar{U}_{\mathrm{TSt}} & =\frac{1}{2} B_{00} c_{22}\left(u_{2}^{(1)}\right)^{2}+\frac{1}{2} B_{11} \bar{c}_{66}\left(u_{2,1}^{(1)}\right)^{2} ; \\
\bar{U}_{\mathrm{TT}} & =\frac{1}{2} B_{00} c_{44}\left(u_{3}^{(1)}\right)^{2}+\frac{1}{2} B_{11} \bar{c}_{55}\left(u_{3,1}^{(1)}\right)^{2} ; \\
\bar{U}_{\mathrm{E}} & =\frac{1}{2} B_{00} c_{11}\left(u_{1,1}^{(0)}\right)^{2} ; \\
\bar{U}_{\mathrm{F}} & =\frac{1}{2} B_{00} c_{66}\left(u_{2,1}^{(0)}\right)^{2} ; \\
\bar{U}_{\mathrm{FS}} & =\frac{1}{2} B_{00} c_{55}\left(u_{3,1}^{(0)}\right)^{2} .
\end{aligned}
$$

We also integrated the energy densities of Eq. (20) as:

$$
\begin{array}{r}
U_{\mathrm{X}}=\int_{-a}^{a} \int_{-c}^{c} \int_{0}^{T} \bar{U}_{X} \mathrm{~d} x_{1} \mathrm{~d} x_{3} \mathrm{~d} t \\
\mathrm{X}=\mathrm{TSh}, \mathrm{TSt}, \mathrm{TT}, \mathrm{E}, \mathrm{F}, \mathrm{FS}
\end{array}
$$

$$
\begin{aligned}
U_{\mathrm{TSh}}= & \frac{2 \pi c}{\omega} c_{66}\left\{\sum_{i=1}^{6} A_{4 i}^{2} \alpha_{4 i}^{2} V_{c 2}\right. \\
& \left.+\sum_{i=1, j \neq i}^{6} A_{4 i} A_{4 j} \alpha_{4 i} \alpha_{4 j} V_{\mathrm{cc}}\right\} \\
& +\frac{\pi^{3} c}{6 \omega} \bar{c}_{11}\left\{\sum_{i=1}^{6} A_{4 i}^{2} \alpha_{4 i}^{2} Z_{i}^{2} V_{s 2}\right. \\
& \left.+\sum_{i=1, j \neq i}^{6} A_{4 i} A_{4 j} \alpha_{4 i} \alpha_{4 j} Z_{i} Z_{j} V_{\mathrm{ss}}\right\}
\end{aligned}
$$




$$
\begin{aligned}
& U_{T \mathrm{St}}=\frac{2 \pi c}{\omega} c_{22}\left\{\sum_{i=1}^{6} A_{4 i}^{2} \alpha_{5 i}^{2} V_{c 2}\right. \\
& \left.+\sum_{i=1, j \neq i}^{6} A_{4 i} A_{4 j} \alpha_{5 i} \alpha_{5 j} V_{\mathrm{cc}}\right\} \\
& +\frac{\pi^{3} c}{6 \omega} \bar{c}_{66}\left\{\sum_{i=1}^{6} A_{4 i}^{2} \alpha_{5 i}^{2} Z_{i}^{2} V_{s 2}\right. \\
& P_{\mathrm{X}}=\frac{U_{\mathrm{X}}}{\tilde{U}} \times 100 \% \text {, } \\
& \mathrm{X}= \begin{cases}\text { TSh,F, } & \text { AT - cut, two modes, } \\
\text { TSh,F,FS, } & \text { AT - cut, three modes, } \\
\text { TSh,TT, TSt, E, F, FS, } & \text { SC - cut, six modes }\end{cases}
\end{aligned}
$$$$
\left.+\sum_{i=1, j \neq i}^{6} A_{4 i} A_{4 j} \alpha_{5 i} \alpha_{5 j} Z_{i} Z_{j} V_{\mathrm{ss}}\right\} \text {; }
$$$$
U_{T T}=\frac{2 \pi c}{\omega} c_{44}\left\{\sum_{i=1}^{6} A_{4 i}^{2} \alpha_{6 i}^{2} V_{c 2}\right.
$$$$
\left.+\sum_{i=1, j \neq i}^{6} A_{4 i} A_{4 j} \alpha_{6 i} \alpha_{6 j} V_{\mathrm{cc}}\right\}
$$$$
+\frac{\pi^{3} c}{6 \omega} \bar{c}_{55}\left\{\sum_{i=1}^{6} A_{4 i}^{2} \alpha_{6 i}^{2} Z_{i}^{2} V_{s 2}\right.
$$$$
\left.+\sum_{i=1, j \neq i}^{6} A_{4 i} A_{4 j} \alpha_{6 i} \alpha_{6 j} Z_{i} Z_{j} V_{\mathrm{ss}}\right\} \text {; }
$$$$
U_{E}=\frac{\pi^{3} c}{\omega} c_{11}\left\{\sum_{i=1}^{6} A_{4 i}^{2} \alpha_{1 i}^{2} Z_{i}^{2} V_{c 2}\right.
$$$$
\left.+\sum_{i=1, j \neq i}^{6} A_{4 i} A_{4 j} \alpha_{1 i} \alpha_{1 j} Z_{i} Z_{j} V_{\mathrm{cc}}\right\} \text {; }
$$$$
U_{\mathrm{F}}=\frac{\pi^{3} c}{\omega} c_{66}\left\{\sum_{i=1}^{6} A_{4 i}^{2} \alpha_{2 i}^{2} Z_{i}^{2} V_{c 2}\right.
$$$$
\left.+\sum_{i=1, j \neq i}^{6} A_{4 i} A_{4 j} \alpha_{2 i} \alpha_{2 j} Z_{i} Z_{j} V_{\mathrm{cc}}\right\}
$$$$
U_{\mathrm{FS}}=\frac{\pi^{3} c}{\omega} c_{55}\left\{\sum_{i=1}^{6} A_{4 i}^{2} \alpha_{3 i}^{2} Z_{i}^{2} V_{c 2}\right.
$$$$
\left.+\sum_{i=1, j \neq i}^{6} A_{4 i} A_{4 j} \alpha_{3 i} \alpha_{3 j} Z_{i} Z_{j} V_{\mathrm{cc}}\right\} \text {; }
$$$$
U=\int_{-a}^{a} \int_{-c}^{c} \int_{0}^{T} \bar{U} \mathrm{~d} x_{1} \mathrm{~d} x_{3} \mathrm{~d} t
$$$$
=U_{\mathrm{TSh}}+U_{\mathrm{TSt}}+U_{\mathrm{TT}}+U_{E}+U_{\mathrm{F}}+U_{\mathrm{FS}} \text {. }
$$

\subsubsection{Energy percentages of each vibration modes}

With all the expressions of strain energies of the plate obtained, we can calculate the energy proportions of each vibration mode. In this study, in order to develop a procedure based on the energy for mode identification, we focus not only on the where $\tilde{U}$ is total of the strain energy of the plate which excludes the coupling energy parts as:

$$
\tilde{U}=\left\{\begin{array}{c}
U_{\mathrm{TSh}}+U_{\mathrm{F}} \\
\mathrm{AT}-\text { cut, two modes } \\
U_{\mathrm{TSh}}+U_{\mathrm{F}}+U_{\mathrm{FS}} \\
\mathrm{AT}-\text { cut, three modes } \\
U_{\mathrm{TSh}}+U_{\mathrm{TSt}}+U_{\mathrm{TT}}+U_{E}+U_{\mathrm{F}}+U_{\mathrm{FS}} \\
\mathrm{SC}-\text { cut, six modes }
\end{array}\right.
$$

while $P_{\mathrm{X}}$ are the percentage of strain energy of the $\mathrm{X}$ mode. The strain energies of coupled modes are neglected due to their insignificance. Such description will be enough for the measurement of dominance of strain energies of each vibration mode.

Since the kinetic energy in vibration also spread to all modes just as the strain energy, and its equivalence to strain energy also implies the possibility to use its proportions for vibration mode identification. With the successful utilization of the strain energy, we also want to explore the possibility and applicability of kinetic energy in a similar manner.

\subsection{The Kinetic Energy}

With known vibrations of the plate, we can calculate the kinetic energy and their proportions as we have demonstrated in the calculation of strain energy. We are expecting that the proportions of kinetic energy are consistent with the strain enshapes.

The kinetic energy of the first-order Mindlin plate is ${ }^{8}$

$$
\begin{aligned}
\bar{K} & =\frac{1}{2} \sum_{m} \sum_{n} \rho B_{\mathrm{mn}} \dot{u}_{j}^{(m)} \dot{u}_{j}^{(n)} \\
& =\frac{1}{2} \rho B_{00} \sum_{i=1}^{3} \dot{u}_{i}^{(0)} \dot{u}_{i}^{(0)}+\frac{1}{2} \rho B_{11} \sum_{i=1}^{3} \dot{u}_{i}^{(1)} \dot{u}_{i}^{(1)} .
\end{aligned}
$$

By separating the vibration displacements as for the calculation of the strain energy, Eq. (24) can also be rewritten by the vibration modes as:

$$
\begin{aligned}
\bar{K}_{\mathrm{TSh}} & =\frac{1}{2} \rho B_{11} \dot{u}_{1}^{(1)} \dot{u}_{1}^{(1)} ; \\
\bar{K}_{\mathrm{TSt}} & =\frac{1}{2} \rho B_{11} \dot{u}_{2}^{(1)} \dot{u}_{2}^{(1)} ; \\
\bar{K}_{\mathrm{TT}} & =\frac{1}{2} \rho B_{11} \dot{u}_{3}^{(1)} \dot{u}_{3}^{(1)} ; \\
\bar{K}_{\mathrm{E}} & =\frac{1}{2} \rho B_{00} \dot{u}_{1}^{(0)} \dot{u}_{1}^{(0)} ; \\
\bar{K}_{\mathrm{F}} & =\frac{1}{2} \rho B_{00} \dot{u}_{2}^{(0)} \dot{u}_{2}^{(0)} ; \\
\bar{K}_{\mathrm{FS}} & =\frac{1}{2} \rho B_{00} \dot{u}_{3}^{(0)} \dot{u}_{3}^{(0)} ;
\end{aligned}
$$
ergies for the determination of dominance of specific mode 
where $\dot{u}_{j}^{(n)}(n=0,1 ; j=1,2,3$,$) are the velocities of each$ mode in vibrations. We can see from Eq. (25), in comparison with the strain energy, the kinetic energy density equations are not coupled. This is certainly advantageous over the strain energies in their complexity for calculation.

With displacement solutions in Eq. (7), we also integrate Eq. (25) over the plate for the three cases of couplings.

\subsubsection{AT-cut quartz crystal plate with two vibration modes}

For two modes only thickness-shear and flexural mode are calculated here as:

$$
\begin{aligned}
K_{\mathrm{X}} & =\int_{-a}^{a} \int_{-c}^{c} \int_{0}^{T} \bar{K}_{\mathrm{X}} \mathrm{d} x_{1} \mathrm{~d} x_{3} \mathrm{~d} t, \mathrm{X}=\mathrm{TSh}, \mathrm{F} ; \\
K_{\mathrm{TSh}} & =\frac{4 \pi b^{2} \rho c}{3 \omega}\left\{\sum_{i=1}^{2} A_{4 i}^{2} \alpha_{4 i}^{2} V_{c 2}+\sum_{i=1, j \neq i}^{2} A_{4 i} A_{4 j} \alpha_{4 i} \alpha_{4 j} V_{\mathrm{cc}}\right\} ; \\
K_{\mathrm{F}} & =\frac{4 \pi b^{2} \rho c}{\omega}\left\{\sum_{i=1}^{2} A_{4 i}^{2} \alpha_{2 i}^{2} V_{s 2}+\sum_{i=1, j \neq i}^{2} A_{4 i} A_{4 j} \alpha_{2 i} \alpha_{2 j} V_{\mathrm{ss}}\right\} \\
K & =\int_{-a}^{a} \int_{-c}^{c} \int_{0}^{T} \bar{K} \mathrm{~d} x_{1} \mathrm{~d} x_{3} \mathrm{~d} t=K_{\mathrm{TSh}}+K_{\mathrm{F}} .
\end{aligned}
$$

\subsubsection{AT-cut quartz crystal plate with three vibration modes}

The kinetic energy of thickness-shear, flexural, and faceshear mode are as:

$$
\begin{aligned}
K_{\mathrm{X}} & =\int_{-a}^{a} \int_{-c}^{c} \int_{0}^{T} \bar{K}_{X} \mathrm{~d} x_{1} \mathrm{~d} x_{3} \mathrm{~d} t, \mathrm{X}=\mathrm{TSh}, \mathrm{F}, \mathrm{FS} ; \text { (27a) } \\
K_{\mathrm{TSh}} & =\frac{4 \pi b^{2} \rho c}{3 \omega}\left\{\sum_{i=1}^{3} A_{4 i}^{2} \alpha_{4 i}^{2} V_{c 2}+\sum_{i=1, j \neq i}^{3} A_{4 i} A_{4 j} \alpha_{4 i} \alpha_{4 j} V_{\mathrm{cc}}\right\}
\end{aligned}
$$

$K_{\mathrm{F}}=\frac{4 \pi b^{2} \rho c}{\omega}\left\{\sum_{i=1}^{3} A_{4 i}^{2} \alpha_{2 i}^{2} V_{s 2}+\sum_{i=1, j \neq i}^{3} A_{4 i} A_{4 j} \alpha_{2 i} \alpha_{2 j} V_{\mathrm{ss}}\right\}$

$K_{\mathrm{FS}}=\frac{4 \pi b^{2} \rho c}{\omega}\left\{\sum_{i=1}^{3} A_{4 i}^{2} \alpha_{3 i}^{2} V_{s 2}+\sum_{i=1, j \neq i}^{3} A_{4 i} A_{4 j} \alpha_{3 i} \alpha_{3 j} V_{\mathrm{ss}}\right\}$

$K=\int_{-a}^{a} \int_{-c}^{c} \int_{0}^{T} \bar{K} \mathrm{~d} x_{1} \mathrm{~d} x_{3} \mathrm{~d} t=K_{\mathrm{TSh}}+K_{\mathrm{F}}+K_{\mathrm{FS}}$

\subsubsection{SC-cut quartz crystal plate with six vibration modes}

In an SC-cut plate all the kinetic energies of the six modes are considered as:

$K_{\mathrm{X}}=\int_{-a}^{a} \int_{-c}^{c} \int_{0}^{T} \bar{K}_{X} \mathrm{~d} x_{1} \mathrm{~d} x_{3} \mathrm{~d} t, \mathrm{X}=$ TSh,TSt, TT, E, F, FS;

$$
K_{\mathrm{TSh}}=\frac{4 \pi b^{2} \rho c}{3 \omega}\left\{\sum_{i=1}^{6} A_{4 i}^{2} \alpha_{4 i}^{2} V_{c 2}+\sum_{i=1, j \neq i}^{6} A_{4 i} A_{4 j} \alpha_{4 i} \alpha_{4 j} V_{\mathrm{cc}}\right\}
$$

$$
K_{\mathrm{TSt}}=\frac{4 \pi b^{2} \rho c}{3 \omega}\left\{\sum_{i=1}^{6} A_{4 i}^{2} \alpha_{5 i}^{2} V_{c 2}+\sum_{i=1, j \neq i}^{6} A_{4 i} A_{4 j} \alpha_{5 i} \alpha_{5 j} V_{\mathrm{cc}}\right\} ;
$$

$$
\begin{aligned}
& K_{\mathrm{TT}}=\frac{4 \pi b^{2} \rho c}{3 \omega}\left\{\sum_{i=1}^{6} A_{4 i}^{2} \alpha_{6 i}^{2} V_{c 2}+\sum_{i=1, j \neq i}^{6} A_{4 i} A_{4 j} \alpha_{6 i} \alpha_{6 j} V_{\mathrm{cc}}\right\} ; \\
& K_{\mathrm{E}}=\frac{4 \pi b^{2} \rho c}{\omega}\left\{\sum_{i=1}^{6} A_{4 i}^{2} \alpha_{1 i}^{2} V_{s 2}+\sum_{i=1, j \neq i}^{6} A_{4 i} A_{4 j} \alpha_{1 i} \alpha_{1 j} V_{\mathrm{ss}}\right\} ;
\end{aligned}
$$

$$
K_{\mathrm{F}}=\frac{4 \pi b^{2} \rho c}{\omega}\left\{\sum_{i=1}^{6} A_{4 i}^{2} \alpha_{2 i}^{2} V_{s 2}+\sum_{i=1, j \neq i}^{6} A_{4 i} A_{4 j} \alpha_{2 i} \alpha_{2 j} V_{\mathrm{ss}}\right\} ;
$$

$$
K_{\mathrm{FS}}=\frac{4 \pi b^{2} \rho c}{\omega}\left\{\sum_{i=1}^{6} A_{4 i}^{2} \alpha_{3 i}^{2} V_{s 2}+\sum_{i=1, j \neq i}^{6} A_{4 i} A_{4 j} \alpha_{3 i} \alpha_{3 j} V_{\mathrm{ss}}\right\}
$$

$$
\begin{aligned}
K & =\int_{-a}^{a} \int_{-c}^{c} \int_{0}^{T} \bar{K} \mathrm{~d} x_{1} \mathrm{~d} x_{3} \mathrm{~d} t \\
& =K_{\mathrm{TSh}}+K_{\mathrm{TSt}}+K_{\mathrm{TT}}+K_{E}+K_{F}+K_{\mathrm{FS}}
\end{aligned}
$$

\subsubsection{Energy percentages of each vibration modes}

Following the same procedure, the distributions of kinetic energies are also calculated as:

$$
\begin{gathered}
\bar{P}_{X}=\frac{K_{\mathrm{X}}}{\tilde{K}} \times 100 \%, \\
\mathrm{X}= \begin{cases}\mathrm{TSh}, \mathrm{F}, & \text { AT - cut, two modes } \\
\text { TSh,F,FS, } & \text { AT - cut, three modes, } \\
\text { TSh,TT, TSt, E, F, FS, } & \text { SC - cut, six modes }\end{cases}
\end{gathered}
$$




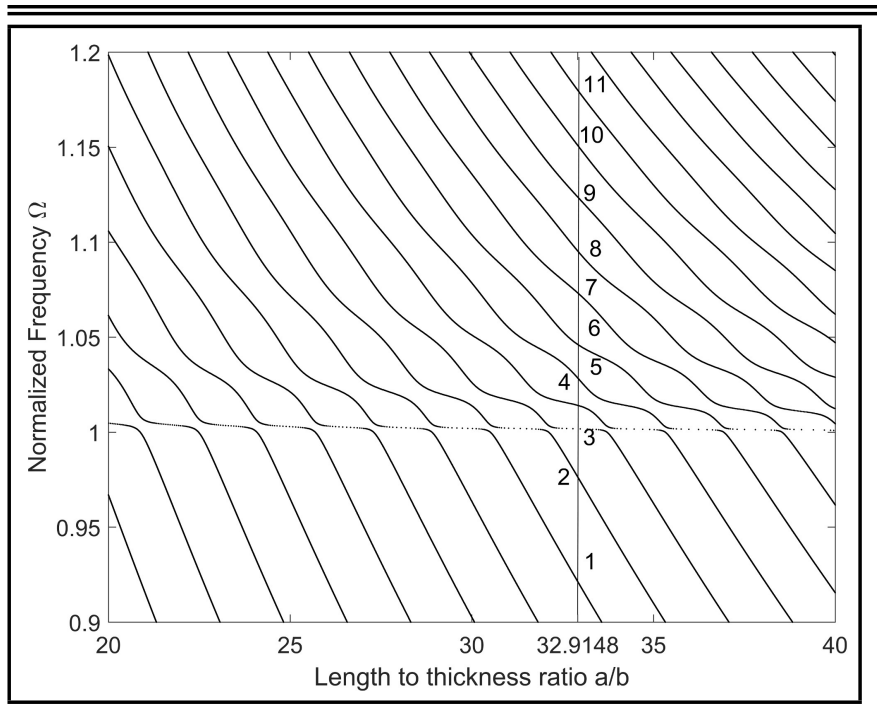

Figure 5. Frequency spectra of two vibration modes and specific length to thickness ratio with 11 resonant frequencies.

Table 1. Strain/Kinetic(S/K) energy distributions of the crystal plate at each resonance with two vibration modes.

\begin{tabular}{||l|l|l|l||}
\hline $\begin{array}{l}\text { Serial } \\
\text { Number }\end{array}$ & $\begin{array}{l}\text { Normalized } \\
\text { Frequency }\end{array}$ & $\begin{array}{l}\text { Flexural } \\
(\text { S/K \% })\end{array}$ & $\begin{array}{l}\text { Thickness-shear } \\
(S / K \%)\end{array}$ \\
\hline $\mathbf{1}$ & 0.9213 & $\mathbf{8 6 . 7 6 / 8 8 . 8 7}$ & $13.24 / 11.13$ \\
$\mathbf{2}$ & 0.9764 & $\mathbf{8 5 . 5 9 / 8 4 . 1 7}$ & $14.41 / 15.83$ \\
$\mathbf{3}$ & 1.0017 & $2.45 / 1.05$ & $\mathbf{9 7 . 5 5 / 9 8 . 9 5}$ \\
$\mathbf{4}$ & 1.0141 & $25.04 / 12.46$ & $\mathbf{7 4 . 9 6 / 8 7 . 5 4}$ \\
$\mathbf{5}$ & 1.0285 & $\mathbf{6 9 . 6 7 / 5 2 . 8 6}$ & $30.33 / 47.14$ \\
$\mathbf{6}$ & 1.0461 & $47.71 / 28.26$ & $\mathbf{5 2 . 2 9 / 7 1 . 7 4}$ \\
$\mathbf{7}$ & 1.0733 & $57.04 / 37.54$ & $\mathbf{4 2 . 9 6 / 6 2 . 4 6}$ \\
$\mathbf{8}$ & 1.0953 & $67.3 / 49.13$ & $32.7 / 50.87$ \\
$\mathbf{9}$ & 1.1237 & $61.75 / 42.49$ & $38.25 / 57.51$ \\
$\mathbf{1 0}$ & 1.1509 & $\mathbf{6 8 . 4 9 / 5 0 . 4 8}$ & $31.51 / 49.52$ \\
$\mathbf{1 1}$ & 1.1800 & $\mathbf{6 8 . 5 7 / 5 0 . 4 9}$ & $31.43 / 49.51$ \\
\hline
\end{tabular}

where $\tilde{K}$ is the sum of the kinetic energy of all vibration modes as:

$$
\tilde{K}=\left\{\begin{array}{c}
K_{\mathrm{TSh}}+K_{\mathrm{F}} \\
\mathrm{AT}-\text { cut, two modes } \\
K_{\mathrm{TSh}}+K_{\mathrm{F}}+K_{\mathrm{FS}} \\
\mathrm{AT}-\text { cut, three modes } \\
K_{\mathrm{TSh}}+K_{\mathrm{TSt}}+K_{\mathrm{TT}}+K_{\mathrm{E}}+K_{\mathrm{F}}+K_{\mathrm{FS}} \\
\mathrm{SC}-\text { cut, six modes }
\end{array}\right.
$$

and $\bar{P}_{X}$ are the proportions of kinetic energies of $\mathrm{X}$ mode. The clear advantage of the kinetic energies is that there is no coupling and the calculation can be simpler.

\section{NUMERICAL RESULTS}

With formulations of energy proportions in terms of displacements, we presented a simple model for energy-based method for the identification of vibration modes in a quartz crystal plate in the vicinity of thickness-shear vibrations. With solutions of displacements and frequencies, the calculations of both strain and kinetic energies are straightforward, and the identification of vibration modes are also done with the numerical value corresponding to each displacement.



Figure 6. Displacements of two modes at a normalized frequency of 1.0017.

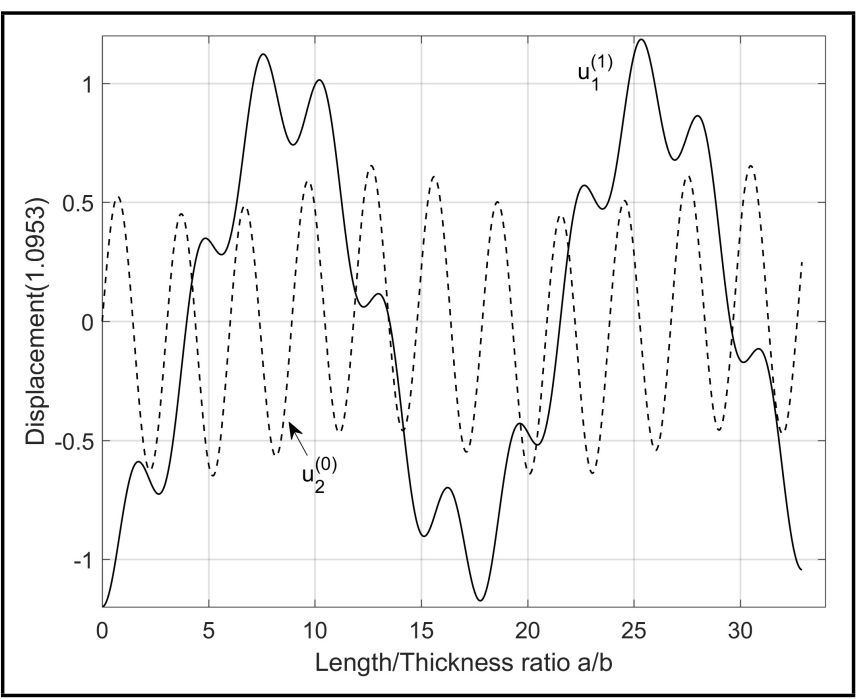

Figure 7. Displacements of two modes at a normalized frequency of 1.0953.

\subsection{AT-cut quartz crystal plate with two vibration modes}

The results have been tabulated in Table 1 for an AT-cut quartz crystal plate with given aspect ratios and thickness for the plate model shown in Fig. 1. The model plate has four free sides and we choose the length to thickness ratio $(a / b)$ to 32.9148 for demonstration purposes. With the given aspect ratio, 11 modes in the frequency spectra and the identification solutions will be applied to these points shown in Fig. 5.

We use Eqs. (23) and (29) for the calculation and the energy distributions of each frequency and the results are given in Table 1.

We also use the displacement data of some frequencies above and plot a few selected mode shapes below for comparisons with the results of Table 1, as shown in Figs. 6 and 7.

From Table 1, it can be seen that the energy distribution has a series of fluctuations and the results of strain energy are similar to the kinetic energy in a systematic trend with small deviations which are mainly caused by the coupling parts of the strain energy at some frequencies. The working frequency are successfully identified as the third frequency with the normal- 


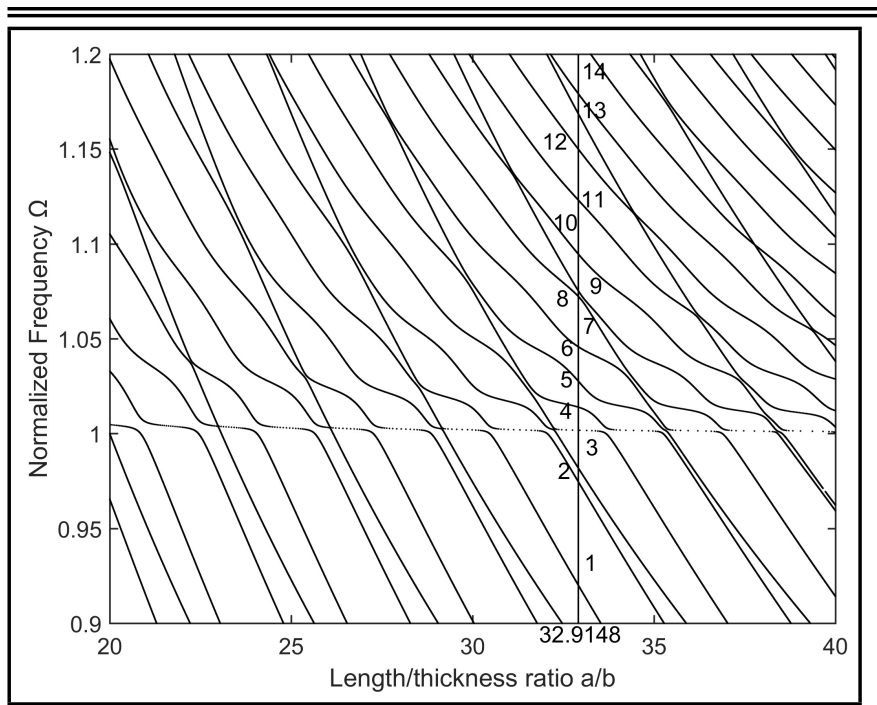

Figure 8. Frequency spectra of three vibration modes and specific length to thickness ratio of 14 resonant frequencies.

Table 2. Strain/Kinetic(S/K) energy distributions of the crystal plate at each resonance with three vibration modes.

\begin{tabular}{|l|l|l|l|l||}
\hline $\begin{array}{l}\text { Serial } \\
\text { Number }\end{array}$ & $\begin{array}{l}\text { Normalized } \\
\text { Frequency }\end{array}$ & $\begin{array}{l}\text { Flexural } \\
(\text { S/K } \%)\end{array}$ & $\begin{array}{l}\text { Face-shear } \\
(\text { S/K \% })\end{array}$ & $\begin{array}{l}\text { Thickness-shear } \\
(S / K \%)\end{array}$ \\
\hline $\mathbf{1}$ & 0.9200 & $\mathbf{7 6 . 4 / 8 8 . 6}$ & $0.4 / 0.4$ & $23.2 / 11.0$ \\
$\mathbf{2}$ & 0.9745 & $\mathbf{7 2 . 0 / 7 8 . 9}$ & $3.9 / 6.4$ & $24.1 / 14.6$ \\
$\mathbf{3}$ & 0.9818 & $9.2 / 5.5$ & $\mathbf{9 7 . 2 / 9 8 . 4}$ & $3.0 / 1.0$ \\
$\mathbf{4}$ & 1.0017 & $2.0 / 1.6$ & $0.0 / 0.0$ & $\mathbf{9 9 . 4 / 9 8 . 4}$ \\
$\mathbf{5}$ & 1.0139 & $17.7 / 13.7$ & $0.1 / 0.1$ & $\mathbf{8 2 . 2 / 8 6 . 2}$ \\
$\mathbf{6}$ & 1.0276 & $\mathbf{5 3 . 9 / 5 3 . 4}$ & $0.3 / 0.3$ & $45.7 / 46.2$ \\
$\mathbf{7}$ & 1.0457 & $29.1 / 26.1$ & $0.2 / 0.1$ & $\mathbf{7 0 . 7 / 7 3 . 8}$ \\
$\mathbf{8}$ & 1.0723 & $39.3 / 36.0$ & $13.5 / 13.3$ & $\mathbf{5 0 . 5 / 5 0 . 7}$ \\
$\mathbf{9}$ & 1.0752 & $5.0 / 3.9$ & $\mathbf{7 7 . 7 / 8 1 . 3}$ & $17.3 / 14.8$ \\
$\mathbf{1 0}$ & 1.0946 & $\mathbf{4 9 . 9 / 4 8 . 1}$ & $0.6 / 0.7$ & $49.5 / 51.2$ \\
$\mathbf{1 1}$ & 1.1230 & $44.9 / 42.7$ & $0.2 / 0.1$ & $\mathbf{5 5 . 0 / 5 7 . 2}$ \\
$\mathbf{1 2}$ & 1.1499 & $\mathbf{5 1 . 5 / 4 9 . 7}$ & $0.8 / 0.9$ & $47.4 / 49.3$ \\
$\mathbf{1 3}$ & 1.1685 & $1.5 / 1.0$ & $\mathbf{9 7 . 5 / 9 8 . 5}$ & $1.0 / 0.5$ \\
$\mathbf{1 4}$ & 1.1791 & $\mathbf{5 1 . 7 / 5 0 . 0}$ & $0.4 / 0.3$ & $47.9 / 49.7$ \\
\hline
\end{tabular}

ized frequency of 1.0017, and the thickness-shear vibrations are considered as the dominant mode because of its highest energy proportion with the percentage value of $98 \%$, almost $100 \%$, implying this frequency represents the optimal functioning mode. The conclusion is also validated by the Fig. 10. With different material types, the effect of energy terms of coupled modes can be significant, as shown at some frequencies in Table 1. Consequently, few modes examined in this material type show that the influences of the coupled strain energy parts are big in some rows while the kinetic energy shows their percentages are nearly half to half. Fig. 7 also supports the viewpoint that the strong coupling may cause deviations of strain energy percentages. The kinetic energy solution is more reliable.

\subsection{AT-cut quartz crystal plate with three vibration modes}

The results have been tabulated in Table 2 for an AT-cut quartz crystal plate with three modes and the model is same as the case of two modes. We also chose the length to thickness ratio $(a / b)$ to 32.9148 for demonstration purposes. With the given aspect ratio, 14 frequencies are in the frequency spectra and the identification techniques based on energy will be applied to these points shown in Fig. 8.



Figure 9. Displacements of the three modes at a normalized frequency of 1.0017.



Figure 10. Displacements of the three modes at a normalized frequency of 1.0752 .

Similarly, the energy distributions of each frequency are calculated and listed in the Table 2.

From Table 2, it is clear that the energy distribution has fluctuations and the results of strain energy are similar to the kinetic energy in a systematic trend with only some minor deviations which are mainly caused by the coupling parts of the strain energy. To see the details, we take a look at the 4th frequency with the mode feature which is obviously simple with energy distributions. At the 4th frequency where the normalized frequency is 1.0017 , and the thickness-shear vibration is the dominant mode because of its highest energy proportion at $99 \%$, almost $100 \%$, implying this frequency can be considered as the optimal functioning mode. For the 5th column, we can see that the strain energy distribution is varying from the lowest 1.0 percent to the highest 99.4 percent and we can quickly locate the mode according to these values. Thus, it demonstrated that the energy method can help us quickly and effectively implement the mode identification technique and find the optimal functioning mode.

For further validation, we took the displacement data of each frequency from the above model and plot mode shapes individ- 


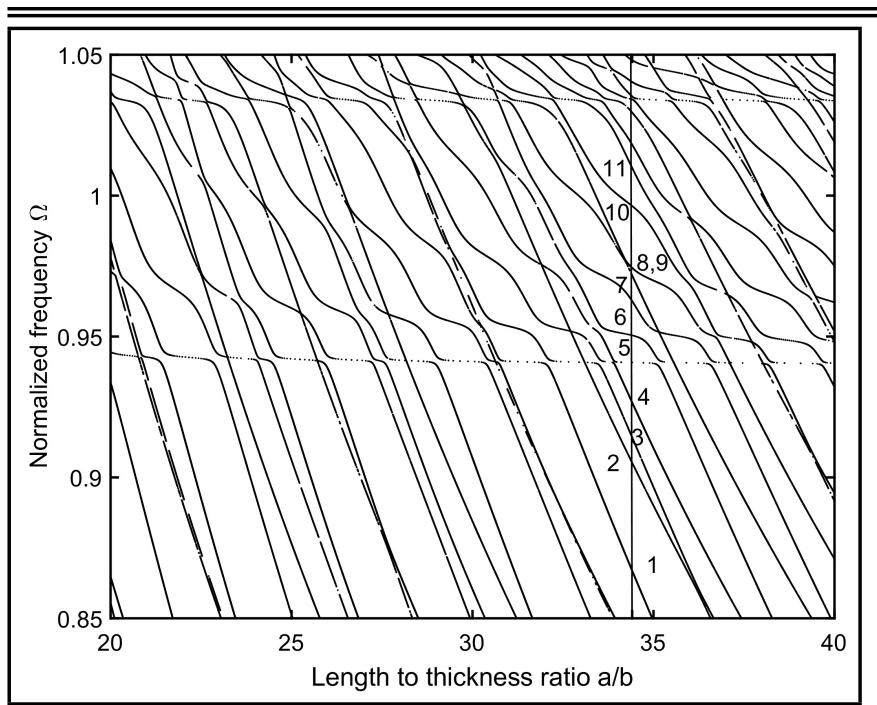

Figure 11. Frequency spectra of six vibration modes and specific length to thickness ratio of 12 resonant frequencies.

ually, as shown in the Figs. 9 and 10.

The mode shapes above clearly show that they are consistent with the findings in Table 2. Taking Fig. 9 as an example, the amplitude of thickness-shear mode has the clear dominance and obviously it is the functioning mode of the thickness-shear vibrations, when its energy distribution reaches $99.4 \%$ in strain and $98.4 \%$ in kinetic energies, respectively. This correlation also applies to other modes and their mode shapes, proving the energy identification method is correct.

\subsection{SC-cut quartz crystal plate with six vibration modes}

The results have been presented in Table 3 for the SC-cut quartz crystal plate and we also chose the length to thickness ratio (a/b) to 34.4097 for demonstration purposes. Meanwhile for the SC-cut plate the frequency of working modes of thickness-shear vibrations are lower than the AT-cut and we chose a different frequency range for the examination. With the given aspect ratio, we chose 12 modes in the frequency spectra and the identification method will be applied to these frequency points as shown in Fig. 11.

By examining the Table 3 , the domain modes are also clearly identified by both strain and kinetic energy solutions. Different from the AT-cut quartz crystal plates above, the concentration of energy of the functioning thickness-shear mode is about $91 \%$ due to more couplings. Some deviations occur when the flexural mode couples with other modes like as in the 4th or 11th frequencies. Other than this, predictions of dominant modes can be trusted based on energy distributions. The further validation of mode shapes are given with Figs. 12 and 13.

From Fig. 12, we find the prediction of mode shapes with kinetic energy pointing to the flexural mode which is clearly the dominant mode. As we know, the SC-cut plate is more likely to be affected by the spurious modes due to stronger couplings. The strain energy solutions are more sensitive to stronger mode couplings as we chose to neglect the coupled terms in strain energies. We can see the kinetic energy is better in determining the feature of strong couplings of vibration modes. In general,

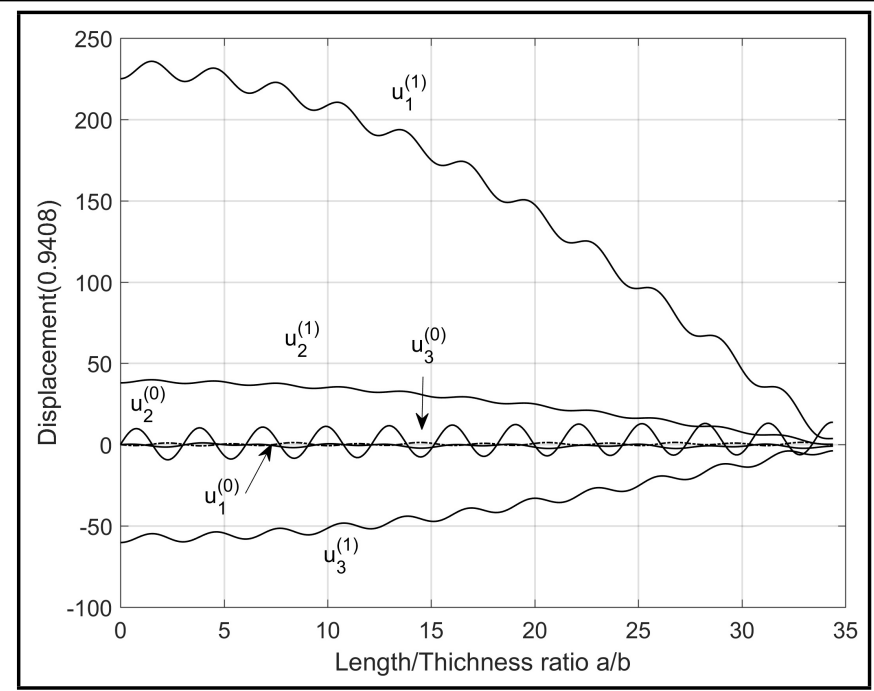

Figure 12. Displacements of the extension and flexural modes from six modes at a normalized frequency of 0.9266 .



Figure 13. Displacements of the six modes at a normalized frequency of 0.9408 .

both methods based on energy are reliable in identifying the dominant vibration modes as shown in Fig. 13.

\subsection{Energy analysis with experimental data}

Since the energy method is accurate with the theoretical analysis, we used the experimental data by Yamashita et al. ${ }^{41}$ for comparison. The models of the experiment are the rectangle AT-cut quartz plates with length-to-thickness aspects from 10.33 to 19.40 and width-to-thickness aspect as 3.78 . We compared the same frequency spectra with mode identification as shown in Fig. 14.

The experimental data marked with " $\mathrm{x}$ " are the thicknessshear modes. The selected four points A, B, C, and D are near the middle of the curves and the dominant thickness-shear modes from the theoretical analysis. ${ }^{15}$ By taking the plate information of the four points, we calculated energies of flexural and thickness-shear modes and list them in Table 4.

In Table 4, the predictions from energy of the mode characters of four points are consistent with the experimental data. The energy values are closer to the $90 \%$ level. It shows the 


\begin{tabular}{|c|c|c|c|c|c|c|c|}
\hline $\begin{array}{l}\text { Serial } \\
\text { Number }\end{array}$ & $\begin{array}{l}\text { Normalized } \\
\text { Frequency }\end{array}$ & $\begin{array}{l}\text { Extension } \\
(S / K \%)\end{array}$ & $\begin{array}{l}\text { Flexural } \\
(S / K \%)\end{array}$ & $\begin{array}{l}\text { Face-shear } \\
(S / K \%)\end{array}$ & $\begin{array}{l}\text { Thickness-stretch } \\
(S / K \%)\end{array}$ & $\begin{array}{l}\text { Thickness-twist } \\
(S / K \%)\end{array}$ & $\begin{array}{l}\text { Thickness-shear } \\
(S / K \%)\end{array}$ \\
\hline 1 & 0.8669 & $0.36 / 2.89$ & 90.39/80.54 & $0.21 / 0.86$ & $0.58 / 1.89$ & $0.15 / 0.32$ & $8.31 / 13.48$ \\
\hline 2 & 0.9052 & $11.18 / 12.88$ & $3.43 / 0.55$ & 78.88/83.05 & $0.15 / 0.11$ & $6 / 3.3$ & $0.37 / 0.11$ \\
\hline 3 & 0.9142 & $1.72 / 15.45$ & 87.79/64.57 & $0.5 / 2.95$ & $0.65 / 1.96$ & $0.17 / 0.34$ & $9.17 / 14.72$ \\
\hline 4 & 0.9266 & $21.56 / 69$ & $65.01 / 15.27$ & $3.55 / 10.15$ & $0.5 / 0.5$ & $0.24 / 0.22$ & $9.13 / 4.87$ \\
\hline 5 & 0.9408 & $0 / 0.01$ & $1.87 / 0.55$ & $0 / 0$ & $2.61 / 5.69$ & $1.82 / 2.6$ & 93.69/91.15 \\
\hline 6 & 0.9504 & $0.09 / 0.27$ & $20.21 / 6.58$ & $0.05 / 0.07$ & $2.29 / 5.6$ & $1.94 / 3.18$ & 75.42/84.29 \\
\hline 7 & 0.9626 & $0.38 / 2.16$ & 74.93/45.42 & $0.21 / 0.6$ & $1.08 / 4$ & $0.75 / 2.33$ & $22.64 / 45.49$ \\
\hline 8 & 0.9717 & $11.2 / 12.63$ & $0.94 / 0.2$ & 78.44/81.03 & $0.22 / 0.22$ & $8.07 / 5.29$ & $1.13 / 0.63$ \\
\hline 9 & 0.9743 & $0.26 / 0.96$ & $55.3 / 24.29$ & $0.31 / 0.76$ & $1.57 / 5.05$ & $1.95 / 4.66$ & $40.61 / 64.28$ \\
\hline 10 & 0.9963 & $0.18 / 0.66$ & $48.95 / 20.2$ & $0.14 / 0.24$ & $1.9 / 5.71$ & $4.16 / 9.08$ & 44.66/64.12 \\
\hline 11 & 1.0099 & $14.16 / 51.71$ & $62.62 / 18.43$ & $2.42 / 7.72$ & $0.97 / 1.78$ & $1.74 / 2.87$ & $18.09 / 17.49$ \\
\hline 12 & 1.0181 & $5.99 / 28.08$ & $64.33 / 24.44$ & $1.28 / 4.58$ & $1.27 / 3.31$ & $4.42 / 9.64$ & $22.7 / 29.95$ \\
\hline
\end{tabular}

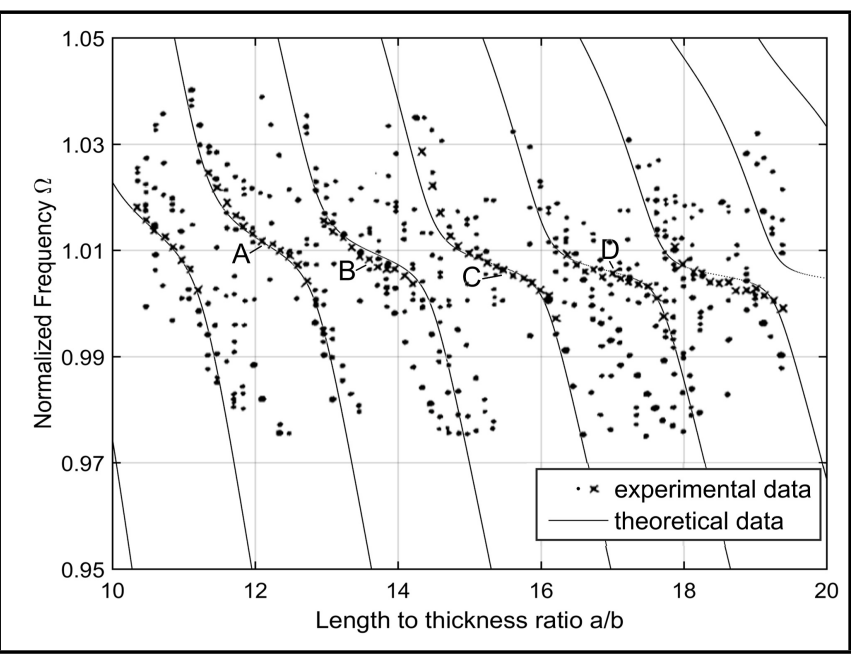

Figure 14. Frequency spectra of the experimental and theoretical data.

Table 4. Strain/Kinetic(S/K) energy distributions of the crystal plate at each resonance with experimental data.

\begin{tabular}{|l|l|l|l|l|l||}
\hline $\begin{array}{l}\text { Expe- } \\
\text { riment } \\
\text { Points }\end{array}$ & $\begin{array}{l}\text { Normalized } \\
\text { Frequency }\end{array}$ & $\begin{array}{l}\text { Length } \\
\text { thickness } \\
\text { ratio }\end{array}$ & $\begin{array}{l}\text { Flexural } \\
(S / K \%)\end{array}$ & $\begin{array}{l}\text { Thickness- } \\
\text { shear } \\
(S / K \%)\end{array}$ & $\begin{array}{l}\text { Dominant } \\
\text { Mode }\end{array}$ \\
\hline $\mathbf{A}$ & 1.01158 & 12.0867 & $14.41 / 6.84$ & $\mathbf{8 5 . 5 9 / 9 3 . 1 6}$ & $\begin{array}{l}\text { Thickness- } \\
\text { shear } \\
\text { Bhickness- } \\
\text { shear } \\
\text { Thickness- } \\
\text { shear } \\
\text { Thickness- } \\
\text { shear }\end{array}$ \\
\hline $\mathbf{C}$ & 1.00833 & 13.5978 & $11.75 / 5.29$ & $\mathbf{8 8 . 2 5 / 9 4 . 7 1}$ \\
\hline
\end{tabular}

consistence of frequency spectra and mode properties of the Mindlin plate theory and the predictions based on energies of vibration modes.

\section{CONCLUSIONS}

The objective of this study was to identify the vibration modes from the coupled vibrations with the Mindlin plate equations at high frequency by means of the energy proportion from displacement solutions. The formulations of kinetic and strain energy densities of the first-order Mindlin plate has been introduced and the total energy is obtained. The vibrations of a simple rectangular AT-cut quartz crystal plate with four free parallel sides were considered without including the electrodes and frequency-temperature effect. The energy distributions of each vibration mode were calculated with the given plate configurations. The comparison between the energy distributions and the mode shape patterns confirmed that the proposed approach actually identified the vibration modes accurately and was able to single out the optimal working mode without using mode shape plots. More importantly, the method was purely numerical and is well suited with the FEM and other numerical solutions. Particularly, using the kinetic energy for the energy distributions of each mode will be a simpler calculation.

Calculating the kinetic and strain energies of the plate within a given frequency range and obtaining the energy distribution of each vibration mode were demonstrated to be simple and easy as exhibited in this study. The conclusion from the energy distribution were consistent with mode shape plots. Although there were some small differences between kinetic and strain energies with the strongly coupled vibration modes, such method of mode identification was simple and reliable. In general, the energy based method for mode identification led to the accurate characterization of vibration modes without difference. Overall this was a convenient and efficient method for the identification of vibration modes with strongly coupled modes and high frequency in structures of anisotropic materials and complex configurations. Furthermore, this energy approach will be particularly preferential in finite element analysis because the energy calculation and characterization can be easily done without utilizing complicated visualization functions and tools. In addition, it was easy to be automated with data flow and the numerical procedure. It is expected that the energy based approach for vibration mode identification can be adopted to meet similar requirements in the finite element analysis of general structures.

\subsection{Acknowledgement}

This work is supported by the National Natural Science Foundation of China (Grants 11672142 and 11772163) and the K. C. Wong Magana Fund through Ningbo University. Additional funding is from the National Key R\&D Program of China (Grant 2017YFB1102900) and the Technology Innovation 2025 Program of the City of Ningbo (Grant 2019B10122).

\section{APPENDIX A}

\section{Equations and parameters for the vibration equations}

The material parameters of quartz crystal and its AT- and SC-cut can be found in Refs. $3,5,8,11,14$ 
The matrix $\mathbf{M}$ and $\mathbf{N}$ in Eq. (6) and Eq. (10) are given for three cases.

1) AT-cut quartz crystal plates with two vibration modes

The dispersion relation from Eq. (6) is given as:

$$
\left|\begin{array}{cc}
Z^{2} \pi\{2\}-12 \Omega^{2} & 2 Z \pi \\
Z \pi & 2\left(\frac{\tilde{c}_{11}}{c_{66}} Z^{2}-\Omega^{2}+1\right)
\end{array}\right|=0 .
$$

The frequency equation in Eq. (10) is given as:

$$
\left|\begin{array}{cc}
\left(\alpha_{22} \frac{\pi Z_{2}}{2}+1\right) \cos \left(\frac{\pi Z_{2}}{2} \frac{a}{b}\right) & \left(\alpha_{24} \frac{\pi Z_{2}}{2}+1\right) \cos \left(\frac{\pi Z_{4}}{2} \frac{a}{b}\right) \\
Z_{2} \sin \left(\frac{\pi Z_{2}}{2} \frac{a}{b}\right) & Z_{4} \sin \left(\frac{\pi Z_{4}}{2} \frac{a}{b}\right)
\end{array}\right|=0 .
$$

2) AT-cut quartz crystal plates with three vibration modes The dispersion relation from Eq. (6) is given as:

$$
\left|\begin{array}{ccc}
\frac{\pi^{2}}{12} Z^{2}-\Omega^{2} & \frac{c_{65}}{c_{66}} \frac{\pi}{2 \sqrt{3}} Z^{2} & \frac{\pi Z}{6} \\
\frac{c_{56}}{c_{66}} \frac{\pi}{2 \sqrt{3}} Z^{2} & \frac{c_{55}}{c_{66}} Z^{2}-\Omega^{2} & \frac{c_{56}}{c_{66}} \frac{Z}{\sqrt{3}} \\
\frac{\pi}{2} Z & \frac{c_{65}}{c_{66}} \sqrt{3} Z & \frac{\tilde{c}_{11}}{c_{66}} Z^{2}-\Omega^{2}+1
\end{array}\right|=0 .
$$

The frequency equation from Eq. (10) is given as:

$$
\begin{aligned}
& \mid\left[\alpha_{32} \frac{\pi Z_{2}}{2}+\kappa_{6} \frac{c_{56}}{c_{55}}\left(\alpha_{22} \frac{\pi Z_{2}}{2}+1\right)\right] \cos \left(\frac{\pi Z_{2}}{2} \frac{a}{b}\right) \\
& {\left[\frac{c_{65}}{c_{66}} \alpha_{32} \frac{\pi Z_{2}}{2}+\kappa_{6}\left(\alpha_{22} \frac{\pi Z_{2}}{2}+1\right)\right] \cos \left(\frac{\pi Z_{2}}{2} \frac{a}{b}\right)} \\
& Z_{2} \sin \left(\frac{\pi Z_{2}}{2} \frac{a}{b}\right) \\
& \begin{array}{c}
{\left[\alpha_{33} \frac{\pi Z_{3}}{2}+\kappa_{6} \frac{c_{56}}{c_{55}}\left(\alpha_{23} \frac{\pi Z_{3}}{2}+1\right)\right] \cos \left(\frac{\pi Z_{3}}{2} \frac{a}{b}\right)} \\
\ldots \quad\left[\frac{c_{65}}{c_{66}} \alpha_{33} \frac{\pi Z_{3}}{2}+\kappa_{6}\left(\alpha_{23} \frac{\pi Z_{3}}{2}+1\right)\right] \cos \left(\frac{\pi Z_{3}}{2} \frac{a}{b}\right) \cdots
\end{array} \\
& Z_{3} \sin \left(\frac{\pi Z_{3}}{2} \frac{a}{b}\right) \\
& \begin{array}{c}
{\left[\alpha_{34} \frac{\pi Z_{4}}{2}+\kappa_{6} \frac{c_{56}}{c_{55}}\left(\alpha_{24} \frac{\pi Z_{4}}{2}+1\right)\right] \cos \left(\frac{\pi Z_{4}}{2} \frac{a}{b}\right)} \\
\cdots\left[\frac{c_{65}}{c_{66}} \alpha_{34} \frac{\pi Z_{4}}{2}+\kappa_{6}\left(\alpha_{24} \frac{\pi Z_{4}}{2}+1\right)\right] \cos \left(\frac{\pi Z_{4}}{2} \frac{a}{b}\right) \mid=0 . \\
Z_{4} \sin \left(\frac{\pi Z_{4}}{2} \frac{a}{b}\right)
\end{array} \mid=
\end{aligned}
$$

3) SC-cut quartz crystal plates with six vibration modes

The dispersion relation from Eq. (6) is given as:

$$
\begin{aligned}
\left|M_{\mathrm{ij}}\right| & =0, i, j=1,2, \ldots 6 ; \\
M_{11} & =\kappa_{1}^{(0)} c_{11} Z^{2}-c_{66} \Omega^{2}, M_{12}=\kappa_{1}^{(0)} \kappa_{6}^{(0)} c_{16} Z^{2}, M_{13} \\
& =\kappa_{1}^{(0)} c_{15} Z^{2} ; \\
M_{14} & =\kappa_{1}^{(0)} \kappa_{6}^{(0)} c_{16} \frac{2}{\pi} Z, M_{15}=\kappa_{1}^{(0)} \kappa_{2}^{(0)} c_{12} \frac{2}{\pi} Z, M_{16} \\
& =\kappa_{1}^{(0)} \kappa_{4}^{(0)} c_{14} \frac{2}{\pi} Z ; \\
M_{21} & =\kappa_{6}^{(0)} c_{61} Z^{2}, M_{22}=\kappa_{6}^{(0)} \kappa_{6}^{(0)} c_{66} Z^{2}-c_{66} \Omega^{2}, M_{23} \\
& =\kappa_{6}^{(0)} c_{65} Z^{2} ; \\
M_{24} & =\kappa_{6}^{(0)} \kappa_{6}^{(0)} c_{66} \frac{2}{\pi} Z, M_{25}=\kappa_{6}^{(0)} \kappa_{2}^{(0)} c_{62} \frac{2}{\pi} Z, M_{26}
\end{aligned}
$$

$$
\begin{aligned}
& =\kappa_{6}^{(0)} \kappa_{4}^{(0)} c_{64} \frac{2}{\pi} Z \\
& M_{31}=\kappa_{5}^{(0)} c_{51} Z^{2}, M_{32}=\kappa_{6}^{(0)} \kappa_{5}^{(0)} c_{56} Z^{2}, M_{33} \\
& =\kappa_{5}^{(0)} c_{55} Z^{2}-c_{66} \Omega^{2} \\
& M_{34}=\kappa_{6}^{(0)} \kappa_{5}^{(0)} c_{56} \frac{2}{\pi} Z, M_{35}=\kappa_{5}^{(0)} \kappa_{2}^{(0)} c_{52} \frac{2}{\pi} Z, M_{36} \\
& =\kappa_{5}^{(0)} \kappa_{4}^{(0)} c_{54} \frac{2}{\pi} Z \\
& M_{41}=\kappa_{6}^{(0)} c_{61} \frac{\pi}{2} Z, M_{42}=\kappa_{6}^{(0)} \kappa_{6}^{(0)} c_{66} \frac{\pi}{2} Z, M_{43} \\
& =\kappa_{6}^{(0)} c_{65} \frac{\pi}{2} Z \\
& M_{44}=\kappa_{6}^{(0)} \kappa_{6}^{(0)}+\frac{\pi^{2}}{12}\left(\kappa_{1}^{(1)} \bar{c}_{11} Z^{2}-\Omega^{2}\right) ; \\
& M_{45}=\frac{\bar{c}_{16}}{3} \kappa_{6}^{(1)} \kappa_{1}^{(1)}\left(\frac{\pi}{2} Z\right)^{2}+c_{62} \kappa_{2}^{(0)} \kappa_{6}^{(0)} \text {; } \\
& M_{46}=\frac{\bar{c}_{15}}{3} \kappa_{1}^{(1)}\left(\frac{\pi}{2} Z\right)^{2}+c_{64} \kappa_{4}^{(0)} \kappa_{6}^{(0)} \\
& M_{51}=\kappa_{2}^{(0)} c_{21} \frac{\pi}{2} Z, M_{52}=\kappa_{2}^{(0)} \kappa_{6}^{(0)} c_{26} \frac{\pi}{2} Z, M_{53} \\
& =\kappa_{2}^{(0)} c_{25} \frac{\pi}{2} Z \\
& M_{54}=\frac{\bar{c}_{61}}{3} \kappa_{6}^{(1)}\left(\frac{\pi}{2} Z\right)^{2}+c_{26} \kappa_{2}^{(0)} \kappa_{6}^{(0)} \text {; } \\
& M_{55}=\frac{\bar{c}_{66}}{3} \kappa_{6}^{(1)} \kappa_{6}^{(1)}\left(\frac{\pi}{2} Z\right)^{2}+c_{22} \kappa_{2}^{(0)} \kappa_{2}^{(0)}-\frac{c_{66}}{3}\left(\frac{\pi}{2}\right)^{2} \Omega^{2} ; \\
& M_{56}=\frac{\bar{c}_{65}}{3} \kappa_{6}^{(1)}\left(\frac{\pi}{2} Z\right)^{2}+c_{24} \kappa_{4}^{(0)} \kappa_{2}^{(0)} \text {; } \\
& M_{61}=\kappa_{4}^{(0)} c_{41} \frac{\pi}{2} Z, M_{62}=\kappa_{4}^{(0)} \kappa_{6}^{(0)} c_{46} \frac{\pi}{2} Z, M_{63}=\kappa_{4}^{(0)} c_{45} \frac{\pi}{2} Z \text {; } \\
& M_{64}=\frac{\bar{c}_{51}}{3} \kappa_{5}^{(1)}\left(\frac{\pi}{2} Z\right)^{2}+c_{46} \kappa_{4}^{(0)} \kappa_{6}^{(0)}, M_{65} \\
& =\frac{\bar{c}_{56}}{3} \kappa_{6}^{(1)} \kappa_{5}^{(1)}\left(\frac{\pi}{2} Z\right)^{2}+c_{42} \kappa_{2}^{(0)} \kappa_{4}^{(0)} \text {; } \\
& M_{66}=\frac{\bar{c}_{55}}{3} \kappa_{5}^{(1)}\left(\frac{\pi}{2} Z\right)^{2}+c_{44} \kappa_{4}^{(0)} \kappa_{4}^{(0)}-\frac{c_{66}}{3}\left(\frac{\pi}{2}\right)^{2} \Omega^{2} \text {. }
\end{aligned}
$$

The frequency equation from Eq. (10) is given as:

$$
\begin{aligned}
\left|N_{\mathrm{jr}}\right|= & 0, j, r=1,2, \ldots 6 ; \\
N_{1 r}= & {\left[\left(c_{61} \alpha_{1 r}+c_{65} \alpha_{3 r}+\kappa_{6}^{(0)} c_{66} \alpha_{2 r}\right) \frac{\pi}{2} Z_{r}+\kappa_{2}^{(0)} c_{62} \alpha_{5 r}\right.} \\
& \left.+\kappa_{4}^{(0)} c_{64} \alpha_{6 r}+\kappa_{6}^{(0)} c_{66} \alpha_{4 r}\right] \cos \left(\frac{\pi Z_{r}}{2} \frac{a}{b}\right) ; \\
N_{2 r}= & {\left[\left(c_{51} \alpha_{1 r}+c_{55} \alpha_{3 r}+\kappa_{6}^{(0)} c_{56} \alpha_{2 r}\right) \frac{\pi}{2} Z_{r}+\kappa_{2}^{(0)} c_{52} \alpha_{5 r}\right.} \\
& \left.+\kappa_{4}^{(0)} c_{54} \alpha_{6 r}+\kappa_{6}^{(0)} c_{56} \alpha_{4 r}\right] \cos \left(\frac{\pi Z_{r}}{2} \frac{a}{b}\right) ; \\
N_{3 r}= & \left(c_{11} \alpha_{1 r}+c_{15} \alpha_{3 r}+\kappa_{6}^{(0)} c_{16} \alpha_{2 r}\right) \frac{\pi}{2} Z_{r}+\kappa_{2}^{(0)} c_{12} \alpha_{5 r} \\
& \left.+\kappa_{4}^{(0)} c_{14} \alpha_{6 r}+\kappa_{6}^{(0)} c_{16} \alpha_{4 r}\right] \cos \left(\frac{\pi Z_{r}}{2} \frac{a}{b}\right) ; \\
N_{4 r}= & \left(\bar{c}_{15} \alpha_{6 r}+\bar{c}_{16} \kappa_{6}^{(1)} \alpha_{5 r}+\bar{c}_{11} \alpha_{4 r}\right) Z_{r} \sin \left(\frac{\pi Z_{r}}{2} \frac{a}{b}\right) ; \\
N_{5 r}= & \left(\bar{c}_{55} \alpha_{6 r}+\bar{c}_{56} \kappa_{6}^{(1)} \alpha_{5 r}+\bar{c}_{51} \alpha_{4 r}\right) Z_{r} \sin \left(\frac{\pi Z_{r}}{2} \frac{a}{b}\right) ;
\end{aligned}
$$


$N_{6 r}=\left(\bar{c}_{65} \alpha_{6 r}+\bar{c}_{66} \kappa_{6}^{(1)} \alpha_{5 r}+\bar{c}_{61} \alpha_{4 r}\right) Z_{r} \sin \left(\frac{\pi Z_{r}}{2} \frac{a}{b}\right)$.

\section{REFERENCES}

1 Du, J., Wang, W., Chen, G., Wu, R., Huang, D., Ma, T., Wang, J., An analysis of thickness-shear vibrations of doubly-rotated quartz crystal plates with the corrected first-order mindlin plate equations, IEEE Transactions on Ultrasonics, Ferroelectrics, and Frequency Control, 60 (11), 2371-2380, (2013). https://dx.doi.org/10.1109/TUFFC.2013.6644740

2 Mindlin, R.D., Spencer, W.J., Anharmonic, thickness-twist overtones of thickness-shear and flexural vibrations of rectangular, AT-cut, quartz plates, Journal of the Acoustical Society of America, 42 (6), 1268-1277, (1967). https://dx.doi.org/10.1109/FREQ.1967.199656

3 Wang, J., Zhao, W., The determination of the optimal length of crystal blanks in quartz crystal resonators, IEEE Transactions on Ultrasonics, Ferroelectrics, and Frequency Control, 52 (11), 2023-2030, (2005). https://dx.doi.org/10.1109/tuffc.2005.1561671

4 Yong, Y.K., Stewart, J.T., Mass-frequency influence surface, mode shapes, and frequency spectrum of a rectangular AT-cut quartz plate, IEEE Transactions on Ultrasonics, Ferroelectrics, and Frequency Control, 38 (1), 67-73, (1991). https://dx.doi.org/10.1109/58.67837

5 Wu, R., Wang, W., Chen, G., Chen, H., Ma, T., $\mathrm{Du}$, J., Wang, J., Free and forced vibrations of SCcut quartz crystal rectangular plates with the first-order Mindlin plate equations, Ultrasonics, 73, 96-106, (2016). https://dx.doi.org/10.1016/j.ultras.2016.09.002

6 Mindlin, R.D., High frequency vibrations of piezoelectric crystal plates, International Journal of Solids and Structures, 8 (7), 895-906, (1972). https://dx.doi.org/10.1016/0020-7683(72)90004-2

7 Wang, J., Yang, J., Higher-order theories of piezoelectric plates and applications, Applied Mechanics Reviews, 53 (4), 87-99, (2000). https://dx.doi.org/10.1115/1.3097341

8 Mindlin, R.D., An Introduction to the Mathematical Theory of Vibrations of Elastic Plates, ed. by Yang, J., World Scientific, Hackensack, NJ, (2006). https://dx.doi.org/10.1142/6309

9 Tiersten, H.F., Mindlin, R.D., Forced vibrations of piezoelectric crystal plates, Quarterly of Appled Mathematics, 20 (2), 107-119, (1962). https://dx.doi.org/10.1090/qam/99964

10 P.C.Y. Lee, Syngellakis, S., Hou, J.P., A two-dimensional theory for high-frequency vibrations of piezoelectric crystal plates with or without electrodes, Journal of Applied Physics, 61 (4), 1249-1262, (1987). https://dx.doi.org/10.1063/1.338102
11 Sekimoto, H., Onozaki, Y., Tamura, T., Goka, S., Watanabe, Y., Two-dimensional analysis of coupled vibrations of UHF AT-cut quartz plates with electrodes of plano-mesa shape, Japanese Journal of Applied Physics, 44 (6b), 4516-4519, (2005)s https://dx.doi.org/10.1143/jjap.44.4516

12 Yong, Y.K., Zhang, Z., Numerical algorithms and results for SC-cut quartz plates vibrating at the third harmonic overtone of thickness shear, IEEE Transactions on Ultrasonics, Ferroelectrics, and Frequency Control, 41 (5), 685-693, (1994). https://dx.doi.org/10.1109/58.308504

13 Wang, J., Yong, Y.K., Imai, T., Finite element analysis of the piezoelectric vibrations of quartz plate resonators with higher-order plate theory, International Journal of Solids and Structures, 36 (15), 2303-2319, (1999). https://dx.doi.org/10.1016/s0020-7683(98)00108-5

14 Yong, Y.K., Wang, J., Imai, T., On the accuracy of mindlin plate predictions for the frequencytemperature behavior of resonant modes in AT- and SCcut quartz plates, IEEE Transactions on Ultrasonics, Ferroelectrics, and Frequency Control, 46 (1), 1-13, (1999). https://dx.doi.org/10.1109/58.741418

15 Wang, J., Hu, W., Zhao, W., Du, J., Huang, D., The finite element analysis of quartz crystal resonators with mindlin plate theory and parallel computing techniques on computer clusters, Proceedings of IEEE Ultrasonics Symposium, New York, NY, USA, 1878-1881, (2007). https://dx.doi.org/10.1109/ultsym.2007.472

16 Wang, J., Chen, L., Du, J., Hu, Y., Li, G., Finite element analysis of nonlinear thickness-shear vibrations of AT-cut quartz crystal plates, Proceedings of IEEE International Frequency Control Symposium, Newport Beach, CA, USA, 392-396, (2010). https://dx.doi.org/10.1109/freq.2010.5556303

17 Wu, R., Wang, J., Du, J., Huang, D., Hu, Y., The non-linear thickness-shear vibrations of quartz crystal plates under an electric field, International Journal of Non-Linear Mechanics, 61, 32-38, (2014). https://dx.doi.org/10.1016/j.ijnonlinmec.2014.01.010

18 Wang, J., Yang, L., Sun, N., Wu, R., Du, J., Huang, D., The fifth-order overtone vibrations of quartz crystal plates with corrected higher-order mindlin plate equations, IEEE Transactions on Ultrasonics Ferroelectrics and Frequency Control, 59 (10), 2278-2291, (2012). https://dx.doi.org/10.1109/tuffc.2012.2453

19 Wu, R., Wang, J., Du, J., Hu, Y., Hu, H., Solutions of nonlinear thickness-shear vibrations of an infinite isotropic plate with the homotopy analysis method, Numerical Algorithms, 59 (2), 213-226, (2012). https://dx.doi.org/10.1007/s11075-011-9485-2

20 Ro, J.J., Baz, A., Optimum placement and control of active constrained layer damping using modal strain energy approach, Journal of Vibration and Control, 8 (6), 861-876, (2002). https://dx.doi.org/10.1177/107754602029204 
${ }^{21}$ He, X., Teh, K., Li, S., Dong, L., Jiang, S., Modeling and experimental verification of an impact-based piezoelectric vibration energy harvester with a rolling proof mass, Sensors and Actuators A: Physical, 259, 171-179, (2017). https://dx.doi.org/10.1016/j.sna.2017.03.034

${ }^{22}$ Shi, J., Fan, C., Zhao, M., Yang, J., Variational formulation of the Stevens-Tiersten equation and application in the analysis of rectangular trapped-energy quartz resonators, The Journal of the Acoustical Society of America, 135 (1), 175181, (2014). https://dx.doi.org/10.1121/1.4829535

23 Yong, Y.K., Patel, M., Tanaka, M., Estimation of quartz resonator $\mathrm{Q}$ and other figures of merit by an energy sink method, IEEE Transactions on Ultrasonics, Ferroelectrics and Frequency Control, 54 (7), 1386-1398, (2007). https://dx.doi.org/10.1109/tuffc.2007.399

${ }^{24}$ Clough, R.W., Penzien, J., Dynamics des structures, New York: McGraw-Hill, (1975).

25 Lyon, R.H., DeJong, R.G., Theory and application of statistical energy analysis, second edition, The Journal of the Acoustical Society of America, 98, 3021, (1995). https://dx.doi.org/10.1121/1.413875

${ }^{26}$ Yan, H., Parrett, A., Nack, W., Statistical energy analysis by finite elements for middle frequency vibration, Finite Elements in Analysis and Design, 35 (4), 297-304, (2000). https://dx.doi.org/10.1016/s0168-874x(99)00071-2

27 Wohlever, J.C., Bernhard, R.J., Mechanical energy flow models of rods and beams, Journal of Sound and Vibration, 153 (1), 1-19, (1992). https://dx.doi.org/10.1016/0022460x(92)90623-6

${ }^{28}$ Wu, Y., Zhang, J., Chen, B., Shen, S., Identification of the dominant vibration modes of single-layer reticulated shells under wind action, International Journal of Space Structures, 22 (2), 123-132, (2009). https://dx.doi.org/10.1260/026635107781482631

${ }^{29}$ Zhong, J., Zhang, J., Zhi, X., Fan, F., Identification of dominant modes of single-layer reticulated shells under seismic excitations, Thin-Walled Structures, 127, 676-687, (2018). https://dx.doi.org/10.1016/j.tws.2018.03.004

30 Ge, M., Zhang, G.C., Du, R., Xu, Y., Feature extraction from energy distribution of stamping processes using wavelet transform, Journal of Vibration and Control, 8 (7), 1023-1032, (2002). https://dx.doi.org/10.1177/107754602029577

31 Wang, Q., Li, Z., Ma, H., Wen, B., Effects of different coupling models of a helical gear system on vibration characteristics, Journal of Mechanical Science and Technology, 31 (5), 2143-2154, (2017). https://dx.doi.org/10.1007/s12206$017-0410-\mathrm{z}$

${ }^{32}$ Huang, Q., Wang, J., Wu, R., Xie, L., Du, J., Using kinetic and strain energies of Mindlin plates for vibration mode identification, Proceedings of
Piezoelectricity, Acoustic Waves, and Device Applications, Chengdu, Sichuan, China, 471-475, (2017). https://dx.doi.org/10.1109/spawda.2017.8340270

33 Wang, J., Yu, J.D., Yong, Y.K., Imai, T., A layerwise plate theory for the vibrations of electroded crystal plates, Proceedings of the 1999 Joint Meeting of the European Frequency and Time Forum and the IEEE International Frequency Control Symposium, Lake Tahoe, NV, USA, 788791, (1999). https://dx.doi.org/10.1109/freq.1999.841423

${ }^{34}$ Wu, R., Wang, J., Du, J., Yang, J., Effects of elliptical ring electrodes on shear vibrations of quartz crystal plates, International Journal of Acoustics and Vibration, 24 (3), 586-591, (2019). https://dx.doi.org/10.20855/10.20855/ijav.2019.24.31405

35 Wang, J., Yu, J.D., Yong, Y.K., Imai, T., A new theory for electroded piezoelectric plates and its finite element application for the forced vibrations of quartz crystal resonators, International Journal of Solids and Structures, 37 (40), 5653-5673, (2000). https://dx.doi.org/10.1016/s00207683(99)00241-3

36 Wang, J., High-Frequency Vibrations of Piezoceramic Plates, in: Hetnarski (Ed.) Encyclopedia of Thermal Stresses, R.B., Springer Netherlands, Dordrecht, pp. 22492253, (2014). https://dx.doi.org/10.1007/978-94-007-27397_132

37 Wang, W.J., Wang, J., Chen, G.J., Du, J.K., Ma, T., Frequency-temperatureanalysis of thickness-shear vibrations of SC-cut quartz crystal plates with the firstorder mindlin plate equations, Proceedings of 2012 Symposium on Piezoelectricity, Acoustic Waves and Device Applications, Shanghai, China, 361-364, (2012). https://dx.doi.org/10.1109/spawda.2012.6464109

38 Wang, J., Yu, J.D., Yong, Y.K., On the correction of the higher-order Mindlin plate theory, International Journal of Applied Electromagnetics and Mechanics, 22 (1-2), 83-96, (2005). https://dx.doi.org/10.3233/jae-2005-693

${ }^{39}$ Mindlin, R.D., Forced thickness-shear and flexural vibrations of piezoelectric crystal plates, Journal of Applied Physics, 23 (1), 83-88, (1952). https://dx.doi.org/10.1063/1.1701983

40 Wang, J., Zhao, W., Bian, T., A fast analysis of vibrations of crystal plates for resonator design applications, Proceedings of the 2004 IEEE International Frequency Control Symposium and Exposition, Montreal, Canada, 596-599, (2004). https://dx.doi.org/10.1109/FREQ.2004.1418527

41 Yamashita, S., Echigo, N., Kawamura, Y., Watanabe, A., Kubota, K., A $4.19 \mathrm{MHz}$ beveled miniature rectangular AT-cut quartz resonator, Proceedings of the 32nd Annual Symposium on Frequency Control, 267-276, (1978). https://dx.doi.org/10.1109/freq.1978.200247 